This is a preprint (not peer reviewed), the first submission to JPO for review was done on 8 Oct 2019.

\title{
Relative Dispersion in the Antarctic Circumpolar Current
}

\author{
DHRUV BALWADA* \\ School of Oceanography, University of Washington, Seattle, Washington, USA \\ JOSEPH H. LACASCE \\ Department of Geosciences, University of Oslo, Oslo, Norway \\ KEVIN G. SPEER \\ Geophysical Fluid Dynamics Institute, Florida State University, Tallahassee, Florida, USA \\ RAFFAELE FERRARI \\ Department of Earth, Atmosphere and Planetary Sciences, Massachusetts Institute of Technology, Cambridge, \\ Massachusetts, USA
}

\begin{abstract}
We present an analysis of relative dispersion and associated metrics from the RAFOS float observations collected during the Diapycnal and Isopycnal Mixing Experiment in the Southern Ocean (DIMES) along with a set of particles from an eddy-resolving numerical model that simulated the flow in the DIMES region. Both RAFOS floats and numerical particles show correlated motions and isotropic pair spreading at length scales smaller than 100-200km and time scales smaller than 50-100 days. Relative dispersion and pair separation PDFs for the RAFOS floats suggest that the stirring the ACC is non-local. While, relative diffusivity, finite scale Lyapunov exponents (FSLEs) and second order structure functions indicate that the stirring might be local. However, these second set of metrics are potentially influenced by internal waves and position errors that do not lead to any cumulative dispersion at timescales longer than a few inertial periods. Thus, the cumulative relative dispersion in the ACC is most probably non-local. Model-particle relative dispersion curves, relative diffusivity, separation PDFs, and FSLEs imply that the stirring experienced by the model particles is non-local, in accordance with the relatively steep energy spectra diagnosed from the model. At large scales the dispersion is anisotropic, with meridional dispersion behaving like random walk and zonal dispersion behaving like shear dispersion. Relative diffusivity from the RAFOS floats and model particles is a function of the separation scales, with values of approximately $O\left(30 \mathrm{~m}^{2} / \mathrm{s}\right)$ at about $10 \mathrm{~km}$ separation, and grows to about $O\left(1000 \mathrm{~m}^{2} / \mathrm{s}\right)$ at $200 \mathrm{~km}$ separations.
\end{abstract}

\section{Introduction}

Oceanic flows are turbulent over a large range of length scales, and are very efficient at stirring tracers and enhancing diffusion by at least 6-7 orders of magnitude over molecular values (Garrett (2006)). This stirring plays an important role in the oceanic transport processes, such as the dispersal of heat, carbon, nutrients and other climatically important tracers (e.g. Gnanadesikan et al. (2015); Fox-Kemper et al. (2013)).

Using observations to characterize the nature of ocean turbulence and to quantify the strength is essential for validating our theoretical framework for modeling these processes, and for helping to guide the parameterizations of

\footnotetext{
* Corresponding author address: Dhruv Balwada, School of Oceanography, University of Washington, Washington, WA, USA.

E-mail: dbalwada@uw.edu
}

unresolved turbulence in circulations models. Lagrangian observations provide one direct way of sampling these turbulent flows, particularly below the surface of the ocean. Different statistical metrics have been devised to convert from raw Lagrangian trajectories to quantitative statements about the underlying flow field. For example "Relative dispersion", the mean square separation of pairs of particles, measures the size of a cluster of particles. The rate of change of dispersion, or eddy diffusivity, quantifies the stirring in a turbulent flow field. Generally, the eddy diffusivity is a function of time and of the scale of the tracer patch, but for a statistically stationary and homogeneous flow, and a tracer patch larger than the dominant eddy scale, the diffusivity asymptotes to a constant. $\mathrm{Nu}-$ merous estimates of ocean diffusivity have been made in this way (Zhurbas and Oh (2003); Koszalka et al. (2011); 
LaCasce et al. (2014); Balwada et al. (2016); Roach et al. $(2016,2018))$.

On the other hand, at smaller length scales, where the velocities are correlated, the diffusivity generally increases with scale (Richardson (1926); Okubo (1971)). Two qualitatively different regimes are possible, non-local and local dispersion (Bennett 1984). Non-local dispersion occurs with steep energy spectra; in this case stirring is dominated by the largest eddies. Under local dispersion, in contrast, stirring is dominated by eddies comparable in scale to the size of the tracer patch.

Observationally characterizing the stirring regime is practically difficult, and requires dense sampling with pairs of instruments, which is why most previous studies have focussed on the surface ocean using surface drifters (LaCasce and Ohlmann 2003; Koszalka et al. 2009; Lumpkin and Elipot 2010; Poje et al. 2014; van Sebille et al. 2015; Beron-Vera and LaCasce 2016; Corrado et al. 2017). The results from such studies have been mixed, with some indicating non-local dispersion up to roughly the deformation scale and others suggesting local dispersion over the same scales. The large scale dispersion varies as well, with some suggesting a transition to diffusive spreading (e.g Koszalka et al. 2009) and other studies suggesting super-diffusive motion, most likely due advection by the large-scale shear (e.g LaCasce and Ohlmann 2003).

Deep ocean studies of stirring, which are rarer, rely on sampling the flow using either an anthropogenic tracer (SF6) (Ledwell et al. 1998; Watson et al. 2013) or RAFOS floats (Rossby et al. 1986). But while a tracer is an excellent means for measuring diapycnal diffusivities (Ledwell et al. 2000; Watson et al. 2013; Ledwell et al. 2016), sampling requirements limit its usefulness for diagnosing lateral stirring on large scales. It is quite useful over small scales though, and results suggest that diffusivities of $\mathrm{O}\left(1-2 \mathrm{~m}^{2} / \mathrm{s}\right)$ on length scales of a few km are typical (Ledwell et al. 1998; Sundermeyer and Ledwell 2001; Shcherbina et al. 2015). Possible causes of the small scale dispersion include internal waves (e.g. Shcherbina et al. 2015), vortical modes (Polzin and Ferrari 2004; Sundermeyer et al. 2005) and coupling between mesoscale vertical shear and vertical diffusion (Haynes 2001; Smith and Ferrari 2009).

RAFOS floats (Swift and Riser 1994) are a good alternative for measuring diffusvities at depth, as their positions are sampled regularly, typically at least once per day. To resolve the smaller scales, the floats need to be deployed in pairs and triplets, which has not routinely been part of float deployment strategies. We are aware of only two previous studies that reported on relative dispersion in the deep ocean (LaCasce and Bower 2000; Ollitrault et al. 2005), both in the North Atlantic Ocean at depths of about $1 \mathrm{~km}$. LaCasce and Bower (2000) concluded the dispersion in the western Atlantic was either local or driven by mean shear up to scales of approximately
$100 \mathrm{~km}$, while the particle pairs separated diffusively in the eastern Atlantic. Ollitrault et al. (2005) also reported local stirring between $40-300 \mathrm{~km}$, and some indications of nonlocal spreading at shorter scales. Non-local stirring yields exponential growth in mean square pair separations, and Ollitrault et al. (2005) suggested an e-folding time scale of 6 days.

We examine observations of stirring at depths of $500-$ $2000 \mathrm{~m}$ in the Southeast Pacific Ocean sector of the Antarctic Circumpolar Current (ACC), using RAFOS floats deployed during the Diapycnal and Isopycnal Mixing Experiment in the Southern Ocean (DIMES) (Balwada et al. 2016). The floats were deployed in pairs and triplets explicitly to resolve small scale dispersion. We resolve length scales of $5-100 \mathrm{~km}$, where the float pair velocities were correlated. This work can be thought of as a further step in the progression of the studies by Tulloch et al. (2014); LaCasce et al. (2014); Balwada et al. (2016), which had reported on the asymptotic eddy diffusivity in the DIMES experiment using both tracer and float observations.

Section 2 describes the data set and some of the specific choices made in our analysis. Section 3, 4, 5 and 6 systematically analyze the data under the lens of several metrics: relative dispersion, pair separation PDFs, finite size Lyapunov exponents and second order longitudinal velocity structure functions. Finally we provide a discussion and conclude in section 7 .

\section{Data}

We examine two sets of Lagrangian trajectories, those from RAFOS floats released during the DIMES experiment (Balwada et al. (2016)), and those from numerical particles advected in a MITgcm simulation of circulation in the Southeast Pacific Ocean and Scotia Sea (LaCasce et al. (2014)).

The DIMES RAFOS floats were released along the $105^{\circ} \mathrm{W}$ meridian and between $54^{\circ}-60^{\circ} \mathrm{S}$ (Figure 1a). The floats behaved akin to isobaric floats and were spread over a depth range of $500-2000 \mathrm{~m}$, with the highest data sampling close to depths of $750 \mathrm{~m}$ and $1400 \mathrm{~m}$. We grouped the RAFOS trajectories into two depth bins: shallow (500$1000 \mathrm{~m})$ and deep (1000-1800m), and only considered segments of the trajectories to the west of $80^{\circ} \mathrm{W}$. The floats rarely came within $100 \mathrm{~km}$ of each other east of this longitude, in the Scotia Sea, and adding them to the analysis produces overly non-homogeneous statistics.

The numerical particles are the same as those used in LaCasce et al. (2014) (Figure 1b). The velocity fields, used to advect the particles, were simulated using the MIT$\mathrm{gcm}$ with a horizontal resolution of $3 \mathrm{~km} \mathrm{X} 6 \mathrm{~km}$ and 70 vertical levels. The model domain spanned $160^{\circ}-20^{\circ} \mathrm{W}$ and $75^{\circ}-35^{\circ} S$, and was forced at the lateral boundaries by the Ocean Comprehensive Atlas (OCCA, Forget (2010)) 
and at the surface by ECMWF ERA-Interim $6 \mathrm{~h}$ wind fields (Berrisford et al. 2009). Details of the model run and comparison to hydrography can be found in Tulloch et al. (2014). 100 particles were released at 20 vertical levels, between $55^{\circ}-60^{\circ} \mathrm{S}$, along $105^{\circ} \mathrm{W}$, at the numerical grid separation of $3 \mathrm{~km}$, every 10 days for 120 days (12 releases). The particles were advected using one day averaged 3D velocity fields, which was sufficient temporal resolution as the energy spectrum drops off sharply $\left(\omega^{-5}\right)$ at periods shorter than about 5 days (not shown). This provided 1200 particle trajectories at each of the 20 levels from $300 \mathrm{~m}$ to $3000 \mathrm{~m}$.

\section{Relative Dispersion}

\section{a. Theory}

Relative dispersion quantifies the spreading of a cluster of particles about its center of mass (Babiano et al. 1990; LaCasce 2008). It is defined as the mean squared separation between particle pairs,

$$
\overline{D^{2}}\left(t, D_{0}\right) \equiv\left\langle\mathbf{D}\left(t, D_{0}\right) \cdot \mathbf{D}\left(t, D_{0}\right)\right\rangle
$$

with $\mathbf{X}\left(\mathbf{a}_{i}, t\right)$ the position of the particle at time $t$ which started at position $\mathbf{a}_{i}$ at $t=0$, while $\mathbf{D}\left(t, D_{0}\right)=\mathbf{D}_{\mathbf{o}}+$ $\left(\mathbf{X}\left(\mathbf{a}_{1}, t\right)-\mathbf{X}\left(\mathbf{a}_{2}, t\right)\right)$, is the separation between a pair with an initial separation of $\mathbf{D}_{0}=\mathbf{a}_{1}-\mathbf{a}_{2},\left(\left|\mathbf{D}_{0}\right|=D_{0}\right)$. The averaging operator $\langle$.$\rangle is conditioned over pairs that are ini-$ tially separated by $D_{0}$, which is indicated as the functional dependence on $D_{0}$. A separation velocity, $\delta \mathbf{V}\left(t, D_{0}\right)=$ $\frac{d}{d t} \mathbf{D}\left(t, D_{0}\right)=\mathbf{u}\left(\mathbf{a}_{1}, t\right)-\mathbf{u}\left(\mathbf{a}_{2}, t\right)$, is the difference between the velocities of the two particles that compose the pair.

The relative diffusivity quantifies the rate of the spreading and is defined as

$$
\begin{aligned}
\kappa\left(t, D_{0}\right) & \equiv \frac{1}{2} \frac{d \overline{D^{2}}\left(t, D_{0}\right)}{d t} \\
& =\left\langle\mathbf{D}\left(t, D_{0}\right) \cdot \delta \mathbf{V}\left(t, D_{0}\right)\right\rangle \\
& =\left\langle\mathbf{D}_{0} \cdot \delta \mathbf{V}\left(t, D_{0}\right)\right\rangle+\int_{0}^{t}\left\langle\delta \mathbf{V}\left(t, D_{0}\right) \cdot \delta \mathbf{V}\left(\tau, D_{0}\right)\right\rangle d \tau .
\end{aligned}
$$

For homogeneous turbulence that is randomly seeded with particles $\left\langle\mathbf{D}_{0} . \delta \mathbf{V}\left(t, D_{0}\right)\right\rangle \approx 0$; the correlation between initial separation and initial relative velocities is by construction small. The covariance in the integral of the equation above can be expanded in terms of the velocities of the individual particles of the pair at time $t$,

$$
\left\langle\delta \mathbf{V}\left(t, D_{0}\right) \cdot \delta \mathbf{V}\left(\tau, D_{0}\right)\right\rangle=2\left\langle\mathbf{u}\left(\mathbf{a}_{1}, t\right) \cdot \mathbf{u}\left(\mathbf{a}_{1}, \tau\right)\right\rangle-2\left\langle\mathbf{u}\left(\mathbf{a}_{1}, t\right)\right.
$$

Homogeneity was used to interchange $\mathbf{a}_{1}$ and $\mathbf{a}_{2}$, when needed, and contract the above expression. If the velocities of pair particles are uncorrelated, this covariance is twice the single particle covariance. At small times, when the pair velocities are perfectly correlated, the relative diffusivity, same as the single particle diffusivity, is a linear function of time and the pairs separate ballistically $\left(\overline{D^{2}} \sim t^{2}\right)$. When the velocities are uncorrelated, after long time and as pairs are farther apart than the largest eddies, the integral in equation 2 converges to a constant, and the relative diffusivity is then twice the single particle (absolute) diffusivity (LaCasce 2008). In this regime the relative dispersion grows linearly $\left(\overline{D^{2}} \sim t\right)$, like brownian motion.

At intermediate times, when the pair velocity is moderately correlated, the diffusivity scales with the particle separation. Scaling relations can then be deduced, on dimensional grounds, following standard turbulence phenomenology. Under the locality hypothesis, the turbulent cascade at a certain scale depends on the local wavenumber $(k)$, for a kinetic energy spectrum $E(k) \propto k^{-\alpha}$

$$
\kappa(D)=\frac{1}{2} \frac{d{\overline{D^{2}}}^{D}}{d t} \propto D^{(\alpha+1) / 2},
$$

where the averaging $\left(\overline{(.)}^{D}\right)$ is performed over all particle pairs at separation of $\mathrm{D}$ initially. A more thorough discussion of this relationship, and test of its validity in idealized simulations can be found in Foussard et al. (2017). Note that the time dependence, present in equation 2 , has been dropped in this relationship. This relationship only holds for values of $1<\alpha \leq 3$ (Bennett 1984), as the locality hypothesis fails for steeper spectra.

The special case of $\alpha=5 / 3$ corresponds to the inverse energy cascade of 2D turbulence (Kraichnan 1967), where the diffusivity is given by "Richardson's law", $\kappa=\beta D^{4 / 3}$ (Richardson 1926), where $\beta$ is proportional to the third root of the energy dissipation rate. For this regime, after an initial period when the memory of the initial condition is lost, the dispersion is (LaCasce 2010):

$$
\overline{D^{2}}=5.2675 \beta^{3} t^{3} .
$$

When $\alpha \geq 3$ (Lin 1972; Lundgren 1981; Bennett 1984), the velocity field at scales smaller than the energy containing scales are smooth and the turbulence is "non-local". Then the diffusivity is $\kappa=D^{2} / T$, where $T$ is proportional to the inverse cubic root of the enstrophy dissipation rate, and the dispersion can be shown to be (Bennett 1984):

$$
\overline{D^{2}}=D_{0}^{2} \exp (8 t / T)
$$

This exponential growth relationship is sometimes also referred to as "Lin's law".

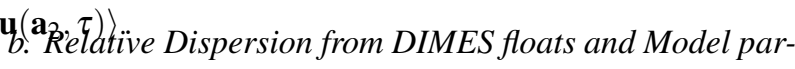 ticles}

We now estimate the relative dispersion that was experienced by the RAFOS floats and the MITgcm particles, and test the theoretical relationships discussed above. 
As the relative dispersion is a function of time $(t)$ and depends on the initial separation $\left(D_{o}\right)$, we considered sets of particle pairs that were initially at different separations. Here we describe how these sets were chosen for the RAFOS floats and the numerical particles.

For the RAFOS floats, only using the pairs that are separated by $D_{0}$ at time of release does not provide a sufficiently large number of samples. So we rely on chance pairs (Morel and Larceveque 1974; LaCasce and Bower 2000) to increase our sample size. Chance pairs are defined as pairs that fortuitously come within a specified separation $\left(D_{0} \pm \delta\right.$, where $\delta$ is a bin size $)$ of each other. The initialization day, day 0 , for the chance pair is chosen to be the first time the pair of floats are within the specified separation bin. If the same pair of floats return to a separation within $D_{0} \pm \delta$ at least 25 days after day 0 , they are further considered as a new pair starting at this new time. However, these instances of pair members converging again are rare. The analysis presented below is for the first 100 days of pair trajectories, as the pair velocities usually get decorrelated within this period. In some instances one or both of the floats might have days with missing data; pairs with less than $25 \%$ sampling during the first 100 days are discarded from the analysis. We found the results to not be sensitive to this choice as long as the vertical separation was kept within reasonable bounds. Most of the analysis presented here for the RAFOS floats corresponds to initial separation bins of $10-15 \mathrm{~km}, 30-35 \mathrm{~km}, 50-55 \mathrm{~km}$. Float pairs were also divided into two sets based on their average depth during the 100 analysis days, shallow set between $500-1000 \mathrm{~m}$ and deep set between $1000-1800 \mathrm{~m}$. We only used pairs in these depth ranges when the two members of the pair were within $100 \mathrm{~m}$ of each other, to minimize the impact of vertical shear on these results.

The pairs in the numerical model were selected by considering trajectories that were initialized at the specific initial separation and at the same depth level. Discrete initial separation categories were possible as the numerical particles were released on a longitude line at the grid separation. Most the analysis presented here corresponds to numerical pairs that were initially separated by $11.1 \mathrm{~km}$, $33.3 \mathrm{~km}$ and $50 \mathrm{~km}$. We focussed on numerical particles that were at depths of $750 \mathrm{~m}$ and $1500 \mathrm{~m}$. The number of samples in the six sets, for both RAFOS floats and model particles, based on initial separation and depth, are shown in Table 1. The choice of six sets is arbitrary but allows us to explore the dependence of the results on depth and initial separation.

The initial separation vector is found to be uncorrelated with the relative velocity, $\left\langle\mathbf{D}_{0} . \delta \mathbf{V}\left(t, D_{0}\right)\right\rangle< \pm 0.2$ at all time (not shown, refer equation 2), for both the RAFOS floats and model particles. This suggests that the choice to consider chance pairs for observations does not introduce any initial correlation between separation velocities and position vector, and is comparable to randomly seeding the flow with particle pairs, as is done for numerical particles.

The analysis here is focused on the range of time and space scales over which the pair velocities are correlated, as uncorrelated motion leads to diffusive dispersion. The analysis for the uncorrelated range of scales was the focus of LaCasce et al. (2014); Balwada et al. (2016); Tulloch et al. (2014). In Figure 2 we show the correlation coefficient between the velocities of the two trajectories that compose the pairs, $\rho=$ $\left\langle\mathbf{u}\left(\mathbf{a}_{1}, t\right) . \mathbf{u}\left(\mathbf{a}_{2}, t\right)\right\rangle /\left\langle\left|\mathbf{u}\left(\mathbf{a}_{1}, t\right)\right|\right\rangle\left\langle\left|\mathbf{u}\left(\mathbf{a}_{2}, t\right)\right|\right\rangle$. Here we discuss the results from the RAFOS floats and model particles in the same vein ${ }^{1}$ as the $\rho$ for the two sets is almost indistinguishable, within errorbars ${ }^{2}$. As expected, $\rho$ reduces as a function of time, and the maximum $\rho$ decreases as a function of initial separation. We also plot $\rho$ as a function of root mean square dispersion $\left(\sqrt{\overline{D^{2}}\left(t, D_{0}\right)}\right)$ (Koszalka et al. 2011; Graff et al. 2015). This choice causes all the correlation curves to approximately collapse on top of each other, suggesting that $\rho$ is a function of only the spatial separation. Finally, we define an associated correlation time scale, as the time when the correlation coefficient drops to a value smaller than 0.5 . The choice of 0.5 is arbitrary; choosing a smaller value would result in somewhat larger correlation scales. Quantification of this time scale gives a rough estimate of how long the correlated motion influences relative dispersion for pairs starting a distance $D_{0}$ apart. We see that this correlation time scale is on the order of 50 days at $D_{0} \sim 10 \mathrm{~km}$ for the deeper set, and on the order of 30 days for the corresponding shallower set. This time scale decreases with depth and with $D_{0}$, and is almost 0 for $D_{0} \sim 60 \mathrm{~km}$. Correspondingly, the spatial scale at which pair velocity correlation drops below 0.5 is about $60-70 \mathrm{~km}$, as can be seen in Figure 2 b,d. A different measure of velocity correlation, as a function of spatial scale, is provided by the second order velocity structure function. This is discussed further in Section 6, and shows that the spatial scale at which the spatial velocity correlation becomes negligible is approximately $\sim 200 \mathrm{~km}$, and is the same as to the distance at which $\rho$ becomes smaller than 0.2 (Figure $2 \mathrm{~b}, \mathrm{~d}$ ). Even with the set of pairs at the smallest initial separations we don't see a period of perfectly correlated pair velocities, and conclude that a ballistic regime is not observed in this data set.

\footnotetext{
${ }^{1}$ In the rest of the results we present the results from the RAFOS floats prior to the model particles, and highlight the start of the individual discussions in italics when appropriate.

${ }^{2}$ All error bars in this study are made by using the bootstrapping algorithm. This involves estimating the metric under question multiple times by creating many different sample sets, and using the $5^{\text {th }}$ and $95^{\text {th }}$ percentiles as the limits of the errorbars. Where each sample set is the same size as the original data set, but derived by performing random draws that allow for repetition. We only show the errorbars in key figures to provide guidance of the level of uncertainty; the errorbars are withheld from some figures to allow for visual clarity.
} 
The relative dispersion as a function of time for different initial separations and different depths, and for the RAFOS floats and model particles, is shown in Figure 3. For the RAFOS floats, the relative dispersion curves, at shallower depth, corresponding to different $D_{0}$ converge to a separation of approximately $300 \mathrm{~km}$ after 100 days. While the relative dispersion, at deeper depth, converged only for the pairs that started at initial separation of $30-35 \mathrm{~km}$ and $50-55 \mathrm{~km}$; the 100 day period was not long enough for the pairs that started out at $10-15 \mathrm{~km}$ to converge with the larger $D_{0}$ sets. Towards the end of period shown in the figure, when pair velocities have decorrelated, most relative dispersion curves portrayed linear growth, in accordance with brownian diffusion $\left(\overline{D^{2}} \sim t\right)$. The relative dispersion from the model particles is in broad agreement with the relative dispersion from the RAFOS floats within error bars, with the agreement being better at deeper than shallower levels.

A decomposition of the relative dispersion into its zonal and meridional components is shown in Figure 4. Since the ACC, the mean flow, is primarily zonal in this region a zonal-meridional decomposition is sufficient to look at the effects of mean flow on relative dispersion. For the RAFOS floats, particularly the deeper sets, the zonal and meridional relative dispersions approximately (visually) isotropic at almost all time shown, isotropy being considered to exist when the zonal and meridional relative dispersions are within an order of magnitude of each other and grow at approximately the same rate. Isotropy is discussed further when presenting relative diffusivity later in this section. The shallow sets show a marked difference in the behavior of the zonal and meridional relative dispersions at late time ( $\sim 30$ days), when the zonal dispersion grows faster than the meridional dispersion. This is presumably a result of the horizontal shear in the mean flow. For the model particles, the zonal relative dispersion is much smaller than the meridional relative dispersion because the model particles were initialized along a longitude line. However, within an order of 10 days the model zonal dispersion catches up with the model meridional dispersion, growing at an approximately ballistic rate. We do not consider this initial difference as a result of anisotropy of the flow dynamics at these scales, but simply a side effect of the initialization. At the shallower depth level the zonal dispersion increases, to exceed the meridional dispersion at longer time, while at the deeper depth level the two remain isotropic for the duration considered here ( $\sim 100$ days). Overall the model particles behave broadly similarly to the RAFOS floats for the decomposed relative dispersions as well. Since the initial phase of the dispersion is isotropic, we can test whether it fits any of the theoretical expectations discussed earlier.

Richardson dispersion predicts a cubic growth asymptotically, equation $5\left(\overline{D^{2}}=5.2675 \beta^{3} t^{3}\right)$, but at initial time this should not be observed due to the dependence on initial separations $\left(D_{0}\right)$. The expression for the evolution during the initial phase is presented in Graff et al. (2015) (Appendix A therein), and appears quite complicated. However, it turns out that a compensated dispersion, $\left(D^{2 / 3}-D_{0}^{2 / 3}\right)$, using Graff's expression has a linear growth, which is easy to compare against a compensated version of the observed dispersion curve. A similar dependence was used in Ollitrault et al. (2005), but the derivation of their theoretical Richardson dispersion at initial time was less rigorous than the one used in Graff et al. (2015). The compensated dispersion from the RAFOS floats and model particles does not show any indication of Richardson dispersion (Figure 3c,d), and the growth rate is faster than the expectation from Richardson dispersion for both the RAFOS floats and the model particles.

If relative dispersion is non-local and follows Lin's law of exponential growth, equation $6\left(\overline{D^{2}}=D_{0}^{2} \exp (8 t / T)\right)$ would hold. This would result in a a straight line on a semilog plot of dispersion. Figure 3 e,f show that on a semilog plot the relative dispersion, for both RAFOS floats and model particles, increases rapidly, possibly exponentially, for the first 10-20 days, and then settles into a slower growth after. This is strongly suggestive of an initial exponential growth, but further confirmation using other metrics is necessary.

The relative diffusivities were calculated using equation 4, where all possible pairs at a certain separation were included in the averaging for the corresponding separation bin, regardless of the initial separation, and averaging was performed after the time derivative was calculated. This is different than the averaging used above, where only pairs within distance $D_{0}$ at $\mathrm{t}=0$ are considered. Prior to calculating the time derivative, which was calculated as the center difference, we smoothed the relative dispersion corresponding to individual pairs using a 2 day running mean. For the RAFOS floats, the relative diffusivity increase as a function of separation, and is isotropic up to approximately $70 \mathrm{~km}$ (Figure 5). The magnitude of relative diffusivity at the shallower depths is slightly greater than the magnitude of relative diffusivity at the deeper levels, at all separations. In the isotropic regime, the diffusivity from the RAFOS floats follows a power law that is close to $4 / 3$, in disagreement with the suggestion of non-locality from the relative dispersion being exponential. The flattening of the observed relative diffusivity curve at scales smaller than $\sim 5 \mathrm{~km}$ might be a result of noise in RAFOS float tracking at the smallest scales. The relative diffusivity from the model particles clearly follows a power law of $r^{2}$, and confirms that the relative dispersion is non-local. The meridional diffusivity for the deep model particles is slightly smaller than the zonal diffusivity.

At large scales, above $200 \mathrm{~km}$, the meridional relative diffusivity, from both RAFOS floats and model particles, saturates to a constant value that is in agreement with the 
results of LaCasce et al. (2014); Balwada et al. (2016), which focused on the diffusivity at the uncorrelated scales of motion. At the same scales, the zonal diffusivity does not saturate, for both RAFOS floats and model particles, and keeps increasing with separation. This growth in zonal diffusivity is presumably a result of the meridional shear in the ACC, and follows a power law of $4 / 3$ in accordance with shear dispersion (LaCasce 2008).

In summary, at the scales of correlated pair velocities the relative dispersion from the RAFOS floats appears to be non-local, while the relative diffusivity follows a power law of $4 / 3$ and suggests local dispersion. This discrepancy could result due to a number of reasons. A) Relative diffusivity magnifies the influence of small scale position perturbations in the trajectory, which are present due to internal waves or position tracking errors in RAFOS float trajectories (see the Lagrangian frequency spectrum discussed below). This influence of the high frequency motions is magnified in the relative diffusivity metric because a time derivative is taken over 2 days. This small scale jiggling will not impact the relative dispersion on time scales longer than a few inertial periods (Beron-Vera and LaCasce 2016) because it generates zero cumulative dispersion, but can impact the results in relative diffusivity like metrics on scales up to 20-60 times larger than the scales of the position perturbations (Haza et al. 2014). B) Alternatively, observed non-local relative dispersion is the result of chance sampling: insufficient sampling for relative dispersion, since we condition pairs based on initial separation, could lead to an emergence of non-local behavior that would disappear if a larger dataset was available. Dräger-Dietel et al. (2018) showed that relative dispersion could portray local or non-local behavior if a drifter data set was appropriately sub-sampled. The model particles, in contrast, show consistent non-local dispersion using both relative dispersion and relative diffusivity.

We believe that the difference in the results between the RAFOS floats and model particles is a result of the higher levels of kinetic energy present at higher frequencies in the RAFOS floats. To highlight this, we consider the Lagrangian frequency spectrum of the velocities measured by the RAFOS floats and model particles (Figure 6), to get an estimate of the kinetic energy contained at different temporal scales. The velocities were estimated by applying center difference on the position data, for both RAFOS floats and model particles. For succinctness, we averaged the spectra from RAFOS trajectories at all depths, and only considered the model particles at $900 \mathrm{~m}$, which is approximately the median depth of the RAFOS floats.

The main result is that the velocities measured by RAFOS floats have a lot more high frequency variability, periods shorter than 10 days, than the model particles; while the kinetic energy at time scales of over 20 days are comparable. Correspondingly, the RAFOS floats shows a marked range that follows a power law of $\omega^{-3}$, while the corresponding scales in the model spectrum has a much steeper power law of $\omega^{-5}$. It is also interesting to note that both RAFOS floats and model particles show a slight preference for cyclonic motions over anticyclonic motions.

The greater kinetic energy present at higher frequencies in RAFOS float velocities supports hypothesis A: the discrepancy between relative dispersion and relative diffusivity estimates from the RAFOS floats is a result of high frequency variability that does not impact relative disperion at time scales longer than a few interial periods. The lower kinetic energy and steeper spectrum present in the model is to be expected, as the model velocity fields are a result of primarily an enstrophy cascade and the presence of a viscous range that can influence motions on scales up to 4-5 times the grid scale (Balwada et al. 2018; Sinha et al. 2019).

\section{Probability Density Functions of the Pair Separa- tions}

\section{a. Theory}

Relative dispersion is the second moment of the pair separation probability density function (PDF). This PDF is expected to be non-gaussian at short separations where velocities are correlated, and thus the second moment is not a complete statistical descriptor of the PDF. Richardson (1926) proposed that the pair separation PDF would evolve according the Focker-Plank equation,

$$
\frac{\partial}{\partial t} p(r, t)=\frac{1}{r} \frac{\partial}{\partial r}\left(r \kappa \frac{\partial}{\partial r} p\right)
$$

where $p(r, t)$ is the pair separation $(r)$ PDF. This equation can be solved exactly for given initial condition, boundary conditions and an expression for relative diffusivity $(\kappa(r))$. LaCasce (2010); Graff et al. (2015) derived solutions assuming that the initial condition is a delta function $\left(p(r, 0)=\frac{1}{2 \pi r} \delta\left(r-D_{o}\right)\right)$, i.e. all particle pairs are released at the same initial separation, and the boundary conditions at $r \rightarrow \infty$ are of no flux. They considered three canonical forms of $\kappa(r) ; \kappa(r)=\beta r^{4 / 3}$ corresponding to Richardson dispersion, which is a particular case of local dispersion, $\kappa(r)=r^{2} / T$ corresponding to non-local dispersion, and a constant $\kappa(r)$ corresponding to the scale independent diffusion, sometimes referred to as Rayleigh dispersion.

We do not reproduce the expressions for the PDF solutions here as they are quite complicated and do not provide direct insight, but they can be found in the appendix of Graff et al. (2015). The PDF corresponding to the specific local regime considered by Richardson is referred to as Richardson PDF, for the non-local regime as Lungdren PDF (Lundgren 1981), and for the diffusive regime as Rayleigh PDF. Moving beyond the second moment discussed in the previous section, we now discuss the expected behavior of the normalized 
fourth moment - the kurtosis $\left(K u=<r^{4}>/<r^{2}>^{2}=\right.$ $\left.\left(\int_{0}^{\infty} r^{5} p d r\right) /\left(\int_{0}^{\infty} r^{3} p d r\right)^{2}\right)$. In the Rayleigh regime the $K u$ asymptotes to 2 , while in the Richardson regime the $K u$ asymptotes to 5.6. In the non-local regime the $K u=e^{8 t / T_{L}}$ grows exponentially in time.

One caveat of these solutions, similar to the scaling arguments considered in the previous section, is that it is assumed that the relative diffusivity scaling applies at all scales, while in nature the range of scales where a regime might be applicable is finite and one should not expect any solution to be valid for all time. Additionally, it should also be kept in mind that with sample sizes, as is often the case for pair dispersion experiments, the confidence in estimating the shape of the pdf and the its higher moments is quite low. Higher moments are harder to estimate, in particular the odd-moments, which is why we only consider the kurtosis in the analysis below.

\section{b. Separation PDFs from DIMES floats and Model Parti- cles}

We now present the separation PDFs and kurtosis for the DIMES RAFOS floats and model particles.

To compare the results to the theoretical solutions we need first to estimate the theoretical growth parameters for the Richardson $\operatorname{regime}(\beta)$, non-local regime $(T)$, and constant diffusion $(\kappa)$. We do not assume that any one regime is the best descriptor, but rather estimate the best fit parameters for all of the regimes and then compare the results. The parameter estimation is done be fitting different relative dispersion curves (theoretical second moment function from Graff et al. (2015) - equations A5, A12 and A18 therein) from day 0 to the day when the dispersion is 5 times the initial dispersion (Graff et al. 2015; Beron-Vera and LaCasce 2016), which happens roughly after 10 days (Table 2). We do the fitting for the set of pairs that are initially at the $10-15 \mathrm{~km}$ separation for the DIMES floats and $11 \mathrm{~km}$ separation for the model particles, as these sets remain in the correlated range of scales for the longest duration. This parameter estimation has been used previously in Graff et al. (2015); Beron-Vera and LaCasce (2016).

The estimated parameters are presented in Table 2, and the fitted dispersion curves along with the actual dispersion are shown in the insets of Figure 7. Not surprisingly, none of the three curves corresponding to the three regimes perfectly match the relative dispersion. The nonlocal dispersion curve appears to be the best candidate, similar to the results from the previous section. The constant diffusivity, Rayleigh dispersion, is the worst and the local dispersion, Richardson, is a mediocre fit. We already know from the previous section that constant diffusivity is not a good model for the behavior at the scales under consideration, and we will not discuss this regime further.

The fits of the PDF at the time when the dispersion is 5 times the initial dispersion are shown in Figure 7, and the evolution of the deep PDFs in time along with the evolutions of the theoretical PDFs are shown in Figure 8. For the deep RAFOS floats both Lungdren and Richardson PDFs seem to be good fits to the observed PDF at the fitting time (11 days), and the Lungdren curve is a slightly better fit at 10 and 20 days, again suggesting that the stirring might be non-local. At longer time, 75 days, neither Richardson or Lungdren provide good fits as most pairs have separated out to uncorrelated scales. The results for the shallower set are similar (not shown). For the deep model particles the Lungdren PDF appears to be the best fit (at 11 days), and describes both the peak and the tails of the distribution. The Richardson PDF has a peak that is quite a bit smaller, and a slightly broader tail. The Lungdren PDF also gives a good fit to the evolution of the PDF, at 10 and 20days, while at day 75 the model particle PDF is in between the Richardson and Lungdren curves.

Finally in Figure 9 we plot the evolution of the kurtosis, which describes the evolution of the tails of the PDF, for all the different initial pair separations considered in the previous section. The kurtosis, similar to relative dispersion, for the RAFOS floats and model particles is very similar and usually match within errorbars, which is why we will mostly discuss them together. The kurtosis for all depths, all initial separation, and both RAFOS floats and model particles increases rapidly for first 5-15days; this indicates that the initial delta function like PDF, where all pair separations are similar, is transitioning to a PDF that occupies a much larger range of separations. Generally the kurtosis increases to higher values and stays high for a longer duration for smaller initial separations. This indicates that the collection of pairs that spend more time sampling the correlated range of scales tend to form longer tailed PDFs. For the pairs released at $10-15 \mathrm{~km}$ initial separation, the peak kurtosis value is greater than 5.6 and suggests that non-local stirring was sampled. The the pairs released at larger initial separation do not reach kurtosis values greater than 5.6; presumably because the tails of PDFs start sampling the uncorrelated range of scales before the kurtosis has had time to grow, which retards the growth of these tails.

In summary, the pair separation PDFs for the RAFOS floats suggests that the dispersion is non-local, while the non-locality of stirring for the model particles appears quite robust. This is broadly inline with the results of the relative dispersion from the previous section.

\section{Finite Size Lyapunov Exponents}

\section{a. Theory}

To alleviate the observationally limited sampling associated with trajectory pairs starting at the same initial separation, it is preferable to change the conditioning of the averaging operation and study the increase in separation 
as a function of scale rather than time ${ }^{3}$. Finite Size Lyapunov Exponents (FSLE) measures the average time taken $(\tau(\delta))$ for a pair of particles to grow in separation from scale of $\delta$ to $r \delta$, where $r>1$ (Artale et al. 1997). FSLE is defined as

$$
\lambda(\delta)=\frac{\log (r)}{\langle\tau(\delta)\rangle}
$$

If the kinetic energy spectrum follows a power law of the form $k^{-\beta}$, the FSLE is expected to scale as $\lambda(\delta) \propto$ $\delta^{(\beta-3) / 2}$ (for $\beta<3$ ), for local stirring. For non-local stirring or smooth velocity fields, $\beta>3$, the FSLE converges to a constant. Thus, the FSLE corresponding to Richardson's law would scale as $\delta^{-2 / 3}$, and corresponding to Lin's law would scale as $\delta^{0}$. For uncorrelated diffusive spreading the scaling would be $\delta^{-2}$.

\section{b. FSLE from DIMES floats and Model Particles}

For calculating FSLE we need to identify pairs of particles that come within $\delta$ distance, and then calculate the time it take for them to reach $r \delta$. Here we take $r=1.4$. We varied $r$ and found no dependence of the results in this section on this choice. For the numerical particles we considered trajectories that were released at the same initial depth for identifying pairs. For the RAFOS floats we found pairs by identified trajectories that came within $\delta$ separation, conditioned on being vertically separated by less than $100 \mathrm{~m}$ at this time.

The RAFOS floats were tracked daily, and the output of the model particles was saved daily. This sets an artificial discreteness on the possible values of $\lambda$, which would particularly be an issue at smaller $\delta$ when particle pairs will separate out to $r \delta$ in relatively few time steps. We linearly interpolated the separation time series between the resolved times to get a better estimate of $\lambda$. For the RAFOS float's FSLE the interpolation causes an increase in the value of the FSLE (compare solid and dashed lines in Figure 10), and also changes the power law behavior at smaller scales (Lumpkin and Elipot 2010; LaCasce 2008). This change in power law at small scales is not sensitive to whether the details of the interpolation, whether it is linear or cubic. This linearly interpolation increases the value of FSLE slightly for the model particles, but does not change the power law behavior of FSLE.

The FSLE from the RAFOS floats shows an approximately $-2 / 3$ dependence at scales smaller than $100 \mathrm{~km}$, at both the shallow and deep levels (Figure 10). At scales larger than 100km the FSLE slope becomes steeper, tending towards -2 . The FSLE from the model particles at scales smaller than $100 \mathrm{~km}$ is almost flat, and markedly different from the RAFOS floats. While, at scales greater than $100 \mathrm{~km}$ the FSLE from model particles is almost identical to that from RAFOS floats.

\footnotetext{
${ }^{3}$ Relative diffusivity estimated in section 3 used this averaging too.
}

In summary, the results of this section suggest that the RAFOS floats experienced local stirring, while the stirring of the model particles was akin to non-local stirring at scales smaller than $100 \mathrm{~km}$. At scales greater than $100 \mathrm{~km}$ both sets show behavior similar to diffusive spreading. There is no qualitative difference between the results of the shallow and deep sets, except for the time scales being faster at shallower depth.

FSLE's suggestion of local stirring for RAFOS floats is reminiscent of the suggestion of local stirring by relative diffusivity. This is a result of the fact that both these metrics highlight the spreading rates at fast times; FSLE by accounting for the time taken to expand over a short separation window and relative diffusivity by taking a time derivative. As discussed at the end of section 3, the cumulative dispersion at scales longer than few inertial periods is presumably not impacted by the small scale position variability, and thus it is more appropriate to categorize the effective relative dispersion as non-local.

\section{Longitudinal Second Order Velocity Structure Functions}

\section{a. Theory}

Relative dispersion is the time integrated result of the relative velocity acting in the longitudinal direction, along the position vector connecting the two particles. Longitudinal second order velocity structure function is a statistical measure that quantifies the properties of this relative velocity, defined as

$$
S 2_{l l}(r)=\left\langle(\delta \mathbf{u}(r) . \hat{\mathbf{r}})^{2}\right\rangle
$$

where $\delta \mathbf{u}(r)$ is the velocity difference between two particles separated by distance $\mathrm{r}, \hat{\mathbf{r}}$ is the unit vector connecting these two particles, and the averaging operator is conditioned over all particle pairs that are separated by distance r. The subscript $l$ indicates that we are considering the longitudinal component of the velocity difference.

Bennett (1984); Babiano et al. (1985, 1990) proposed that for an energy spectrum following the power law $k^{-\beta}$ over a long enough range of scales, the structure function followed a power law behavior of $r^{\beta-1}$.

Bennett (1984); Babiano et al. (1990) also show that an exact theoretical relationship to link the relative dispersion to the energy spectrum, and thus the second order structure can be derived (equation 3.6 in Bennett (1984)). However, evaluating this relationship in general is not possible, and progress is made only by assuming power law behavior of the energy spectrum that results from purely vortical turbulence.

Babiano et al. (1990), instead of using the conventional relative dispersion (section 3 ), defined a quantity referred 
to as the instantaneous relative dispersion coefficient

$$
\begin{aligned}
\chi(r) & =\left\langle\left(\frac{1}{2} \frac{d}{d t} \mathbf{D}(t) \cdot \mathbf{D}(t)\right)^{2}\right\rangle_{D}^{1 / 2} \\
& =\left[S 2_{l l}(D)\right]^{1 / 2} D,
\end{aligned}
$$

where $\langle.\rangle_{D}$ is averaging ${ }^{4}$ conditioned when $|\mathbf{D}|=D$, independent of initial pair separation $D_{0}$. The root mean square form of instantaneous relative dispersion coefficient and the form of conditional averaging differentiates it from the regular relative diffusivity considered previously (Eqn 2 and 4). The connection of the instantaneous relative dispersion coefficient to the energy spectrum is quite direct, via the connection to the $S 2_{l l}$, but the connection of relative diffusivity (eqn 4) is based on scaling arguments and long inertial ranges.

We are including the analysis of the $S 2_{l l}$ here for completeness, as numerous recent studies have included this metric along with relative dispersion diagnostics. However, we want to acknowledge that link of the $S 2_{l l}$ to relative dispersion is less direct, and the link is more direct to the less useful instantaneous relative dispersion coefficient. In particular, $S 2_{l l}$ is a good representation of the energy spectrum, which includes influence from internalwaves (LaCasce 2016; Beron-Vera and LaCasce 2016), and similar to relative diffusivity and FSLE might highlight the influence of small-scale perturbations that do not result in cumulative dispersion.

\section{b. S2 ${ }_{l l}$ from DIMES floats and Model particles}

In order to calculate $S 2_{l l}$ we identified trajectories that came within a separation $r$ of each other. We used the same separations bins as those used in the FSLE calculations of the previous section. For numerical particles we considered all trajectories that were initially released as part of the same depth set. For RAFOS floats we divided the trajectories into the two depth groups, and then only considered trajectories that were within $100 \mathrm{~m}$ of each other vertically. We only show results for separation bins that had more than 100 samples.

At scales greater than approximately $100-200 \mathrm{~km} S 2_{l l}$ for both the RAFOS floats and model particles becomes constant, indicating that the spatial velocity correlation between particle pairs at this separation is negligible. At these decorrelated scales $S 2_{l l}$ is an indicator of the eddy kinetic energy (EKE). A comparison of this saturation value between the model and observations suggests that the EKE in the model is slightly smaller than that observed in the DIMES region. The scale at which velocity becomes decorrelated is slightly greater at the shallower depth $(200 \sim 300 \mathrm{~km})$, than at deeper depth $(100 \sim$ $200 \mathrm{~km}$ ). At smaller scales the $S 2_{l l}$ from both the RAFOS

\footnotetext{
${ }^{4}$ same averaging as relative diffusivity and FSLE.
}

floats and model particles is scale dependent, with $S 2_{l l}$ from RAFOS floats having a much shallower slope than then $S 2_{l l}$ from the model particles. The slope of the observational $S 2_{l l}$ is close to $2 / 3$ between scales of 3 to $20 \mathrm{~km}$, and becomes slightly steeper to have slopes close to 1 between 20 and $100 \mathrm{~km}$. While, the slope of the numerical $S 2_{l l}$ is approximately $3 / 2$. Qualitatively the shape of the $S 2_{l l}$ between the shallow and deep observations is similar. We show in a complementary study (Balwada et al 2019 (in prep.)) that the shallow slopes for the observational data are probably a result of inertia-gravity waves, which will not play a role in transport and dispersion (Balwada et al. 2018).

\section{Discussion and Conclusions}

In this study we provided an observational perspective on turbulent stirring in the subsurface Antarctic Circumpolar Current (ACC) at length scales comparable to and smaller than the largest observed eddies $(1-100 \mathrm{~km})$, and is one of the rare observational studies that addresses the nature of sub-mesoscale flows in the subsurface ocean. We examined Lagrangian stirring in the Southeast Pacific Ocean sector of the ACC, a relatively low kinetic energy region, using the DIMES RAFOS float trajectories and particles released in an eddy-resolving $(\triangle x \sim 5 \mathrm{~km})$ MIT$\mathrm{gcm}$ simulation with a variety of metrics.

One of the aims was to categorize if the stirring is local, primarily influenced by eddies that are the size of the pair separation scales, or non-local, primarily influenced by eddies that are much bigger than the pair separation scales. The RAFOS floats indicated that the stirring is non-local, when considering metrics that highlight the cumulative dispersion at time scales longer than few inertial periods. While, metrics that are more sensitive to small-scale perturbations in the trajectory, suggested that the RAFOS float dispersion might be local. However, we believe that this is an artifact of these metric; highlighting high-frequency dynamics that do not produce cumulative dispersion. All metrics indicated that the stirring experienced by the model particles is non-local. This nonlocality observed in the model particles is presumably a result of limited spatial resolution, as the energy spectra in numerical models are quite steep (Figure 6), differing from observations, up to length scales of about 5 times the grid resolution.

Pair member velocities are correlated up to scales of $100-200 \mathrm{~km}$, which corresponds to about 30 and 50 days of correlated motion for the shallow and deep floats that started at initial separations of $10 \mathrm{~km}$. During this time, the relative dispersion grows isotropically, at similar rates in the zonal and meridional direction, up to separations corresponding to the size of the largest eddies, after which the zonal growth becomes faster than the meridional growth. 
The overall rate of separation slows down once the separation scale reaches the size of the largest eddies, evidenced by the convergence of the dispersion curves for different initial separations. The relative dispersion for the RAFOS floats and model particles is broadly consistent. Plots of compensated relative dispersion showed that the the growth rates are more consistent with non-local exponential growth, rather than a Richardson type local growth. The relative diffusivity for the RAFOS floats indicated that the stirring is local, but as discussed at the end of section 3 this is probably a result of internal waves that do not result in any cumulative dispsersion at time scales longer than a few inertial periods. The relative diffusivity for the model particles showed a clear presence of non-local stirring at the correlated scales, which is in agreement with the steep energy spectrum.

At large scales the dispersion is anisotropic, with meridional dispersion behaving like random walk and zonal dispersion behaving like shear dispersion. The meridional relative diffusivity saturated to constant values $O\left(1000 \mathrm{~m}^{2} / \mathrm{s}\right)$ at scales greater than $200 \mathrm{~km}$, which are in agreement with estimates of single particle diffusivity for this region from previous studies (LaCasce et al. 2014; Balwada et al. 2016; Tulloch et al. 2014). This large scale meridional diffusivity is approximately two orders of magnitude larger than the relative diffusivity at scales smaller than $10 \mathrm{~km}$, which is in agreement with the estimates of small scale diffusivity estimates from tracer roughness (Boland et al. 2015).

The relative separation PDF and kurtosis evolution over time suggested the presence of non-local dispersion rather than local dispersion, for both RAFOS floats and model particles. There did not seem to be any systematic differences between the RAFOS floats and model particles for these metric, except that the observational data was more noisy due to limited sample size. These metrics, similar to relative dispersion, are indicators of cumulative dispersion and less sensitive to high frequency perturbations.

The finite size Lyapunov exponent (FSLE) and longitudinal velocity structure function $\left(S 2_{l l}\right)$ indicated a marked difference between the RAFOS floats and the model particles, with both metrics indicating the presence of a more energetic flow field at length scales smaller than $100 \mathrm{~km}$ in the real ACC as compared to the modeled flow. This is also in agreement with the relative diffusivity, which also averages at fixed separation scale, rather than following the evolution of the trajectories in time. However, as has been pointed out by previous studies (Beron-Vera and LaCasce 2016), the interpretation of these metrics is questionable in the presence of inertia-gravity waves. These waves can have strong signatures on the energy spectrum, which is comparable to the $S 2_{l l}$, and other metrics that are sensitive to high frequency dynamics, but do not produce any cumulative dispersion (Balwada et al. 2018). The comparison between the behavior of these two metrics and relative dispersion have only been done in idealized studies simulating a purely turbulent flow, with no wave modes, and thus the biases present in these metrics in the presence of waves are not well documented. Thus, we do not take the indication of local dispersion in these metrics as an absolute guarantee that the dispersion is truly local.

At the end, we want to acknowledge that the observational results have large errorbars due to limited sampling, particularly for metrics that rely on following particle pairs over a long duration (relative dispersion, separation PDFs, and kurtosis). While, we have discounted the suggestion of local stirring by metrics that highlight small and fast scales (relative diffusivity, FSLE, $S 2_{l l}$ ), and believe that these metrics are corrupted only by linear internal waves that do not lead to any cumulative dispersion, it is possible that truth is somewhere in the middle. One cannot entirely discount the possibility that small scale features in the flow might be present that can lead to cumulative dispersion. Such flow dynamics in the deep ocean can potentially result from interaction between internal waves and balanced flows (Thomas and Yamada 2019), or more esoteric flow features referred to as vortical modes, which result due to breaking waves creating mixed patches that then coalesce due to an inverse cascade (Sundermeyer et al. 2005; Polzin and Ferrari 2004). Thus, the case on the true nature of stirring in deep ocean is not closed, and more dedicated observational work needs to be done to untangle these interesting complexities.

Acknowledgments. DB and KS acknowledge support from NSF OCE 1658479 and NSF OCE 1231803. JHL was supported by by the NORSEE project number 221780, under the Norwegian Research Council.

\section{References}

Artale, V., G. Boffetta, A. Celani, M. Cencini, and A. Vulpiani, 1997: Dispersion of passive tracers in closed basins: Beyond the diffusion coefficient. Physics of Fluids, 9 (11), 3162-3171.

Babiano, A., C. Basdevant, P. Le Roy, and R. Sadourny, 1990: Relative dispersion in two-dimensional turbulence. Journal of Fluid Mechanics, 214, 535-557.

Babiano, A., C. Basdevant, and R. Sadourny, 1985: Structure functions and dispersion laws in two-dimensional turbulence. Journal of the atmospheric sciences, 42 (9), 941-949.

Balwada, D., K. S. Smith, and R. Abernathey, 2018: Submesoscale vertical velocities enhance tracer subduction in an idealized antarctic circumpolar current. Geophysical Research Letters, 45 (18), 97909802 .

Balwada, D., K. G. Speer, J. H. LaCasce, W. B. Owens, J. Marshall, and R. Ferrari, 2016: Circulation and stirring in the southeast pacific ocean and the scotia sea sectors of the antarctic circumpolar current. Journal of Physical Oceanography, 46 (7), 2005-2027, doi:10.1175/ JPO-D-15-0207.1, URL http://dx.doi.org/10.1175/JPO-D-15-0207. 1, http://dx.doi.org/10.1175/JPO-D-15-0207.1.

Bennett, A., 1984: Relative dispersion: Local and nonlocal dynamics. Journal of the atmospheric sciences, 41 (11), 1881-1886. 
Beron-Vera, F. J., and J. H. LaCasce, 2016: Statistics of simulated and observed pair separations in the gulf of mexico. Journal of Physical Oceanography, 46 (7), 2183-2199, doi:10.1175/JPO-D-15-0127.1, URL http://dx.doi.org/10.1175/JPO-D-15-0127.1, http://dx.doi.org/ 10.1175/JPO-D-15-0127.1.

Berrisford, P., D. Dee, K. Fielding, M. Fuentes, P. Kallberg, S. Kobayashi, and S. Uppala, 2009: The era-interim archive. ERA report series, (1), 1-16.

Boland, E. J. D., E. Shuckburgh, P. H. Haynes, J. R. Ledwell, M.-J. Messias, and A. J. Watson, 2015: Estimating a submesoscale diffusivity using a roughness measure applied to a tracer release experiment in the southern ocean. Journal of Physical Oceanography, 45 (6), 1610-1631, doi:10.1175/JPO-D-14-0047.1, URL http://dx.doi.org/10.1175/JPO-D-14-0047.1, http://dx.doi.org/ 10.1175/JPO-D-14-0047.1.

Corrado, R., G. Lacorata, L. Palatella, R. Santoleri, and E. Zambianchi, 2017: General characteristics of relative dispersion in the ocean. Scientific Reports, 7, 46291, doi:10.1038/srep46291, URL http://www.ncbi.nlm.nih.gov/pmc/articles/PMC5387742/.

Dräger-Dietel, J., K. Jochumsen, A. Griesel, and G. Badin, 2018: Relative dispersion of surface drifters in the benguela upwelling region. Journal of Physical Oceanography, 48 (10), 2325-2341.

Forget, G., 2010: Mapping ocean observations in a dynamical framework: A 2004-06 ocean atlas. Journal of Physical Oceanography, 40 (6), 1201-1221.

Foussard, A., S. Berti, X. Perrot, and G. Lapeyre, 2017: Relative dispersion in generalized two-dimensional turbulence. Journal of Fluid Mechanics, 821, 358-383.

Fox-Kemper, B., R. Lumpkin, and F. Bryan, 2013: Lateral transport in the ocean interior. Ocean Circulation and Climate: A 21st century perspective, 103, 185-209.

Garrett, C., 2006: Turbulent dispersion in the ocean. Progress in Oceanography, 70 (2), 113-125.

Gnanadesikan, A., M.-A. Pradal, and R. Abernathey, 2015: Isopycnal mixing by mesoscale eddies significantly impacts oceanic anthropogenic carbon uptake. Geophysical Research Letters, 42 (11), 4249-4255, doi:10.1002/2015GL064100, URL file:///Users/dhruvb/ Downloads/ams_phoc36_2232.bib, 2015GL064100.

Graff, L., S. Guttu, and J. LaCasce, 2015: Relative dispersion in the atmosphere from reanalysis winds. Journal of the Atmospheric Sciences, 72 (7), 2769-2785.

Haynes, P. H., 2001: Vertical shear plus horizontal stretching as a route to mixing. Tech. rep., CAMBRIDGE UNIV (UNITED KINGDOM) DEPT OF APPLIED MATHEMATICS AND THEORETICAL PHYSICS.

Haza, A. C., T. M. Özgökmen, A. Griffa, A. C. Poje, and M.-P. Lelong, 2014: How does drifter position uncertainty affect ocean dispersion estimates? Journal of Atmospheric and Oceanic Technology, 31 (12), 2809-2828.

Koszalka, I., J. LaCasce, M. Andersson, K. Orvik, and C. Mauritzen, 2011: Surface circulation in the nordic seas from clustered drifters. Deep Sea Research Part I: Oceanographic Research Papers, 58 (4), 468-485.

Koszalka, I., J. LaCasce, and K. Orvik, 2009: Relative dispersion in the nordic seas. Journal of Marine Research, 67 (4), 411-433.
Kraichnan, R. H., 1967: Inertial ranges in two-dimensional turbulence. Tech. rep., DTIC Document.

LaCasce, J., 2008: Statistics from lagrangian observations. Progress in Oceanography, 77 (1), 1-29.

LaCasce, J., 2010: Relative displacement probability distribution functions from balloons and drifters. Journal of Marine Research, 68 (34), 433-457.

LaCasce, J., 2016: Estimating eulerian energy spectra from drifters. Fluids, 1 (4), 33.

LaCasce, J., and A. Bower, 2000: Relative dispersion in the subsurface north atlantic. Journal of Marine Research, 58 (6), 863-894.

LaCasce, J., and C. Ohlmann, 2003: Relative dispersion at the surface of the gulf of mexico. Journal of Marine Research, 61 (3), 285-312.

LaCasce, J. H., R. Ferrari, J. Marshall, R. Tulloch, D. Balwada, and K. Speer, 2014: Float-derived isopycnal diffusivities in the dimes experiment. Journal of Physical Oceanography, 44 (2), 764-780, doi:10.1175/JPO-D-13-0175.1, URL http://dx.doi.org/10. 1175/JPO-D-13-0175.1, http://dx.doi.org/10.1175/JPO-D-13-0175. 1.

Ledwell, J., E. Montgomery, K. Polzin, L. S. Laurent, R. Schmitt, and J. Toole, 2000: Evidence for enhanced mixing over rough topography in the abyssal ocean. Nature, 403 (6766), 179.

Ledwell, J. R., R. He, Z. Xue, S. F. DiMarco, L. J. Spencer, and P. Chapman, 2016: Dispersion of a tracer in the deep gulf of mexico. Journal of Geophysical Research: Oceans, 121 (2), 1110-1132.

Ledwell, J. R., A. J. Watson, and C. S. Law, 1998: Mixing of a tracer in the pycnocline. Journal of Geophysical Research: Oceans, 103 (C10), 21 499-21 529.

Lin, J.-T., 1972: Relative dispersion in the enstrophy-cascading inertial range of homogeneous two-dimensional turbulence. Journal of the Atmospheric Sciences, 29 (2), 394-396.

Lumpkin, R., and S. Elipot, 2010: Surface drifter pair spreading in the north atlantic. Journal of Geophysical Research: Oceans, 115 (C12).

Lundgren, T. S., 1981: Turbulent pair dispersion and scalar diffusion. 111, 27-57.

Morel, P., and M. Larceveque, 1974: Relative dispersion of constantlevel balloons in the 200-mb general circulation. Journal of the Atmospheric Sciences, 31 (8), 2189-2196.

Okubo, A., 1971: Oceanic diffusion diagrams. Deep sea research and oceanographic abstracts, Elsevier, Vol. 18, 789-802.

Ollitrault, M., C. Gabillet, and A. C. De Verdiere, 2005: Open ocean regimes of relative dispersion. Journal of fluid mechanics, 533, 381407.

Orsi, A. H., T. Whitworth, and W. D. Nowlin, 1995: On the meridional extent and fronts of the antarctic circumpolar current. Deep Sea Research Part I: Oceanographic Research Papers, 42 (5), 641-673.

Poje, A. C., and Coauthors, 2014: Submesoscale dispersion in the vicinity of the deepwater horizon spill. Proceedings of the National Academy of Sciences, 111 (35), 12 693-12 698.

Polzin, K., and R. Ferrari, 2004: Isopycnal dispersion in natre. Journal of physical oceanography, 34 (1), 247-257. 
Richardson, L. F., 1926: Atmospheric diffusion shown on a distanceneighbour graph. Proceedings of the Royal Society of London. Series A, Containing Papers of a Mathematical and Physical Character, 110 (756), 709-737.

Roach, C. J., D. Balwada, and K. Speer, 2016: Horizontal mixing in the southern ocean from argo float trajectories. Journal of Geophysical Research: Oceans, 121 (8), 5570-5586.

Roach, C. J., D. Balwada, and K. Speer, 2018: Global observations of horizontal mixing from argo float and surface drifter trajectories. Journal of Geophysical Research: Oceans.

Rossby, T., D. Dorson, and J. Fontaine, 1986: The rafos system. Journal of Atmospheric and Oceanic Technology, 3 (4), 672-679.

Shcherbina, A., and Coauthors, 2015: The latmix summer campaign: submesoscale stirring in the upper ocean. Bulletin of the American Meteorological Society, 96 (8), 1257-1279.

Sinha, A., D. Balwada, N. Tarshish, and R. Abernathey, 2019: Modulation of lateral transport by submesoscale flows and inertia-gravity waves. Journal of Advances in Modeling Earth Systems, 11 (4), 1039-1065.

Smith, K. S., and R. Ferrari, 2009: The production and dissipation of compensated thermohaline variance by mesoscale stirring. Journal of Physical Oceanography, 39 (10), 2477-2501.

Sundermeyer, M. A., and J. R. Ledwell, 2001: Lateral dispersion over the continental shelf: Analysis of dye release experiments. Journal of Geophysical Research: Oceans, 106 (C5), 9603-9621.

Sundermeyer, M. A., J. R. Ledwell, N. S. Oakey, and B. J. Greenan, 2005: Stirring by small-scale vortices caused by patchy mixing. Journal of physical oceanography, 35 (7), 1245-1262.

Swift, D. D., and S. C. Riser, 1994: Rafos floats: Defining and targeting surfaces of neutral buoyancy. Journal of atmospheric and Oceanic technology, 11 (4), 1079-1092.

Thomas, J., and R. Yamada, 2019: Geophysical turbulence dominated by inertia-gravity waves. Journal of Fluid Mechanics, 875, 71-100.

Tulloch, R., and Coauthors, 2014: Direct estimate of lateral eddy diffusivity upstream of drake passage. Journal of Physical Oceanography, 44 (10), 2593-2616.

van Sebille, E., S. Waterman, A. Barthel, R. Lumpkin, S. R. Keating, C. Fogwill, and C. Turney, 2015: Pairwise surface drifter separation in the western pacific sector of the southern ocean. Journal of Geophysical Research: Oceans, 120 (10), 6769-6781.

Watson, A. J., J. R. Ledwell, M.-J. Messias, B. A. King, N. Mackay, M. P. Meredith, B. Mills, and A. C. N. Garabato, 2013: Rapid crossdensity ocean mixing at mid-depths in the drake passage measured by tracer release. Nature, 501 (7467), 408.

Zhurbas, V., and I. S. Oh, 2003: Lateral diffusivity and lagrangian scales in the pacific ocean as derived from drifter data. Journal of Geophysical Research: Oceans, 108 (C5). 
TABLE 1. Number of pairs at different depths and for different initial separations.

\begin{tabular}{lccc}
\hline Model Trajectories & $11.1 \mathrm{~km}$ & $33.3 \mathrm{~km}$ & $50 \mathrm{~km}$ \\
\hline $750 \mathrm{~m}$ & 1176 & 1128 & 1068 \\
$1500 \mathrm{~m}$ & 1176 & 1128 & 1068 \\
\hline DIMES Trajectories & $10-15 \mathrm{~km}$ & $30-35 \mathrm{~km}$ & $50-55 \mathrm{~km}$ \\
$500-1000 \mathrm{~m}$ & 51 & 85 & 128 \\
$1000-1800 \mathrm{~m}$ & 93 & 188 & 299 \\
\hline
\end{tabular}

\begin{tabular}{c|cccc}
\hline Trajectories & $\beta\left(\mathrm{m}^{2 / 3} /\right.$ day $)$ & $T_{L}$ (days) & $\kappa\left(\mathrm{m}^{2} / \mathrm{s}\right)$ (constant) & $T^{*}$ (days) \\
\hline Model $11 \mathrm{~km}, 750 \mathrm{~m}$ & 28.3 & 30.2 & 216.9 & 7 \\
Model $11 \mathrm{~km}, 1500 \mathrm{~m}$ & 13.5 & 59.9 & 94.1 & 12 \\
DIMES 10-15 km, 500-1000m & 20.8 & 42.7 & 175.3 & 9 \\
DIMES 10-15 km, 1000-18000m & 17.8 & 49.5 & 147.9 & 11
\end{tabular}

TABLE 2. Parameters by fitting second moment solutions to relative dispersion over day 0 to time when the dispersion is 5 times the initial dispersion. $T^{*}$ is the time when the dispersion is 5 times the initial dispersion.
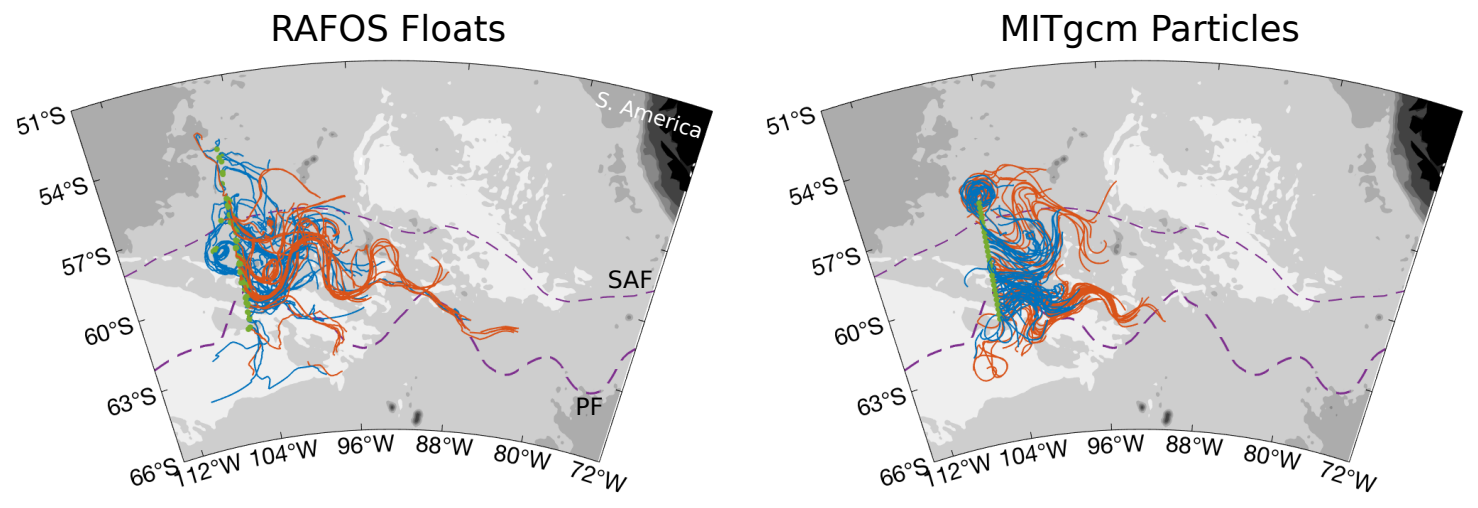

FIG. 1. 100 day trajectories of RAFOS floats (left) and a representative set of numerical particles from the MITgcm simulation (right). Orange trajectories are for shallower depths $(500-1000 \mathrm{~m}$ for RAFOS floats, and MITgcm particles launched at $\sim 750 \mathrm{~m})$, while blue trajectories are for deeper depths $(1000-1800 \mathrm{~m}$ for RAFOS floats, and MITgcm particles launched at $\sim 1500 \mathrm{~m})$. The climatological Sub-Antarctic Front (SAF) and Polar Front (PF) are marked by dashed purple lines (Orsi et al. 1995). The gray colors represent the bathymetry, with the lightest contour color starting at $-6000 \mathrm{~m}$ depth, and increasing by $1000 \mathrm{~m}$ intervals. 


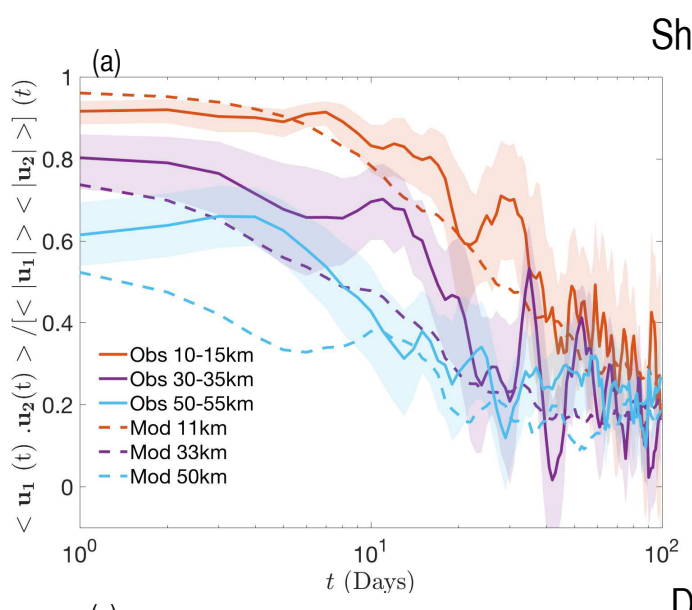

Shallow
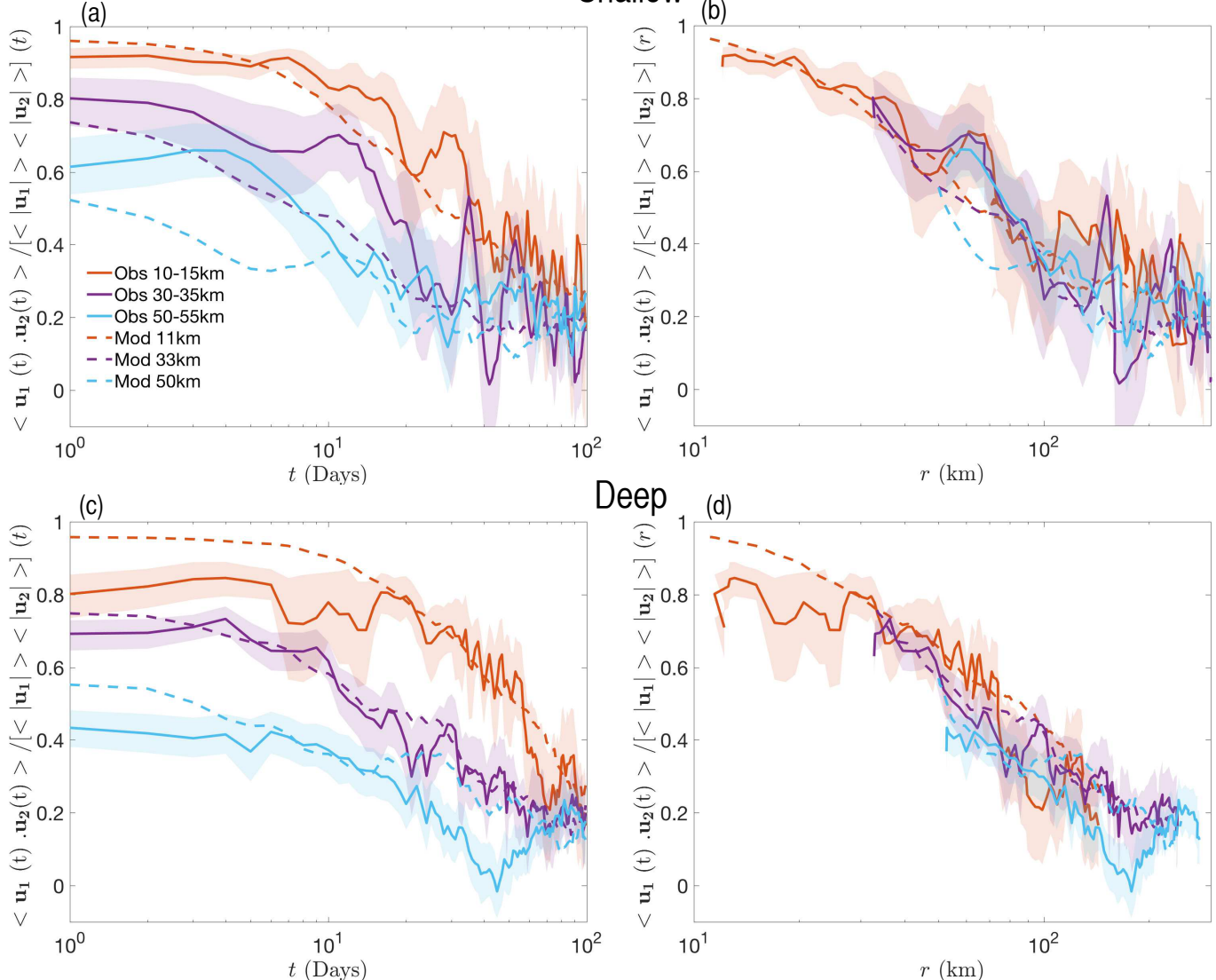

Deep
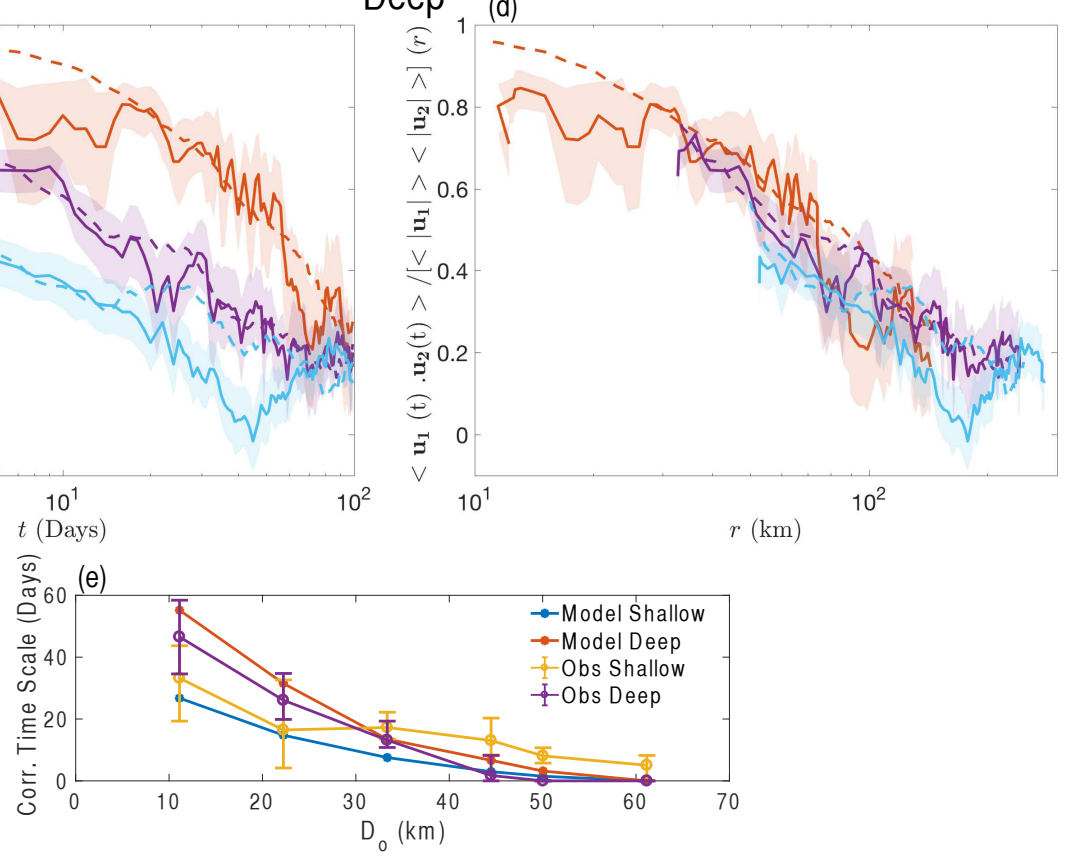

FIG. 2. Correlation of velocities of the two trajectories that compose a pair at shallow and deep levels, as a function of time (a,c) and as a function of spatial scale measured by the root mean square dispersion $(D(t))(\mathrm{b}, \mathrm{d})$. The legend in (a) labels the initial separation ranges and the model/RAFOS trajectories to which each curve corresponds. Dashed lines mark the numerical particles and the solid lines with error bars mark the RAFOS floats. (e) Correlation time scale, time when the correlation curve in (a) or (c) crosses a value of 0.5, as a function of initial separation $\left(D_{0}\right)$. 
(a)

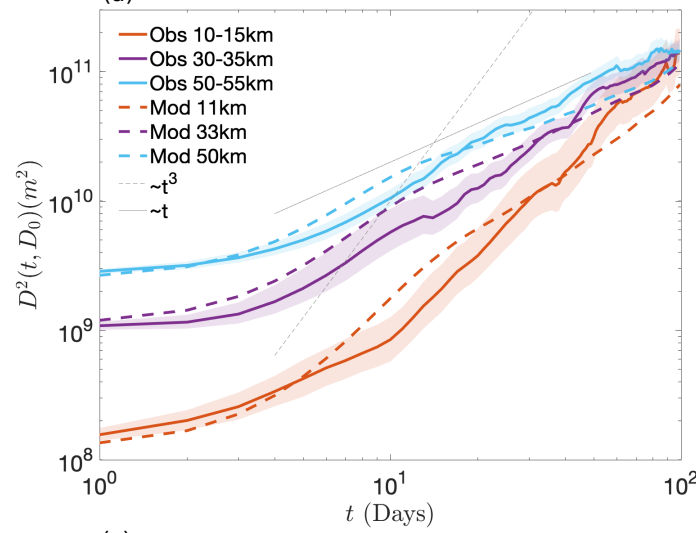

(c)

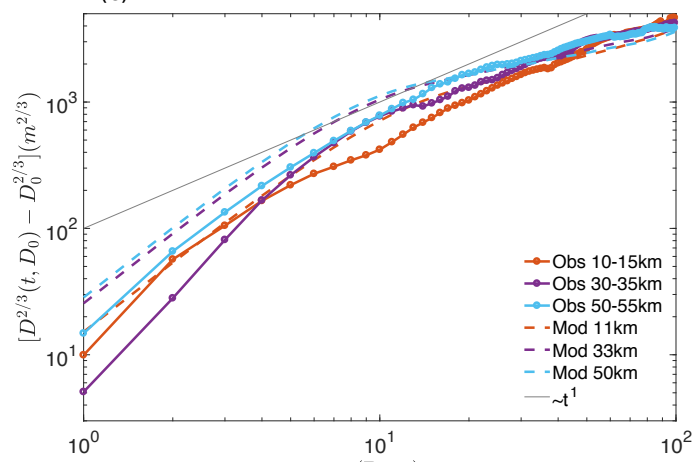

(e)

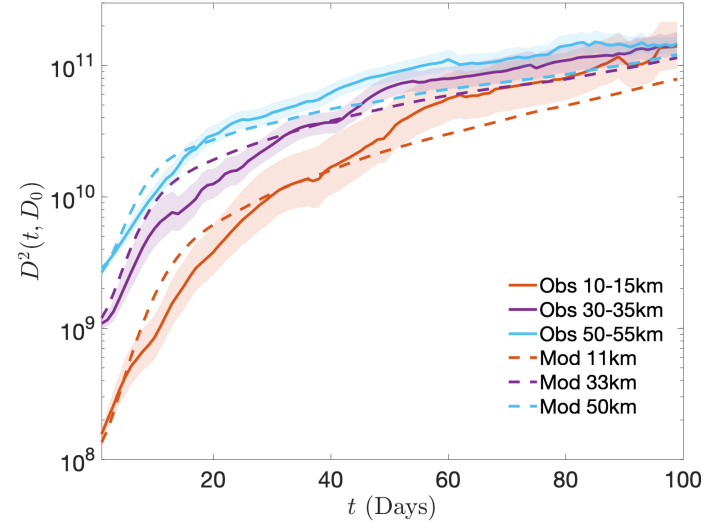

(b) Deep

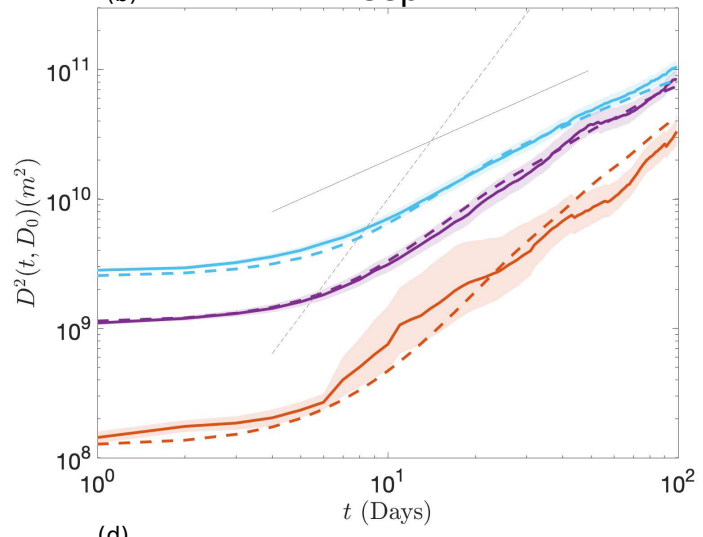

(d)
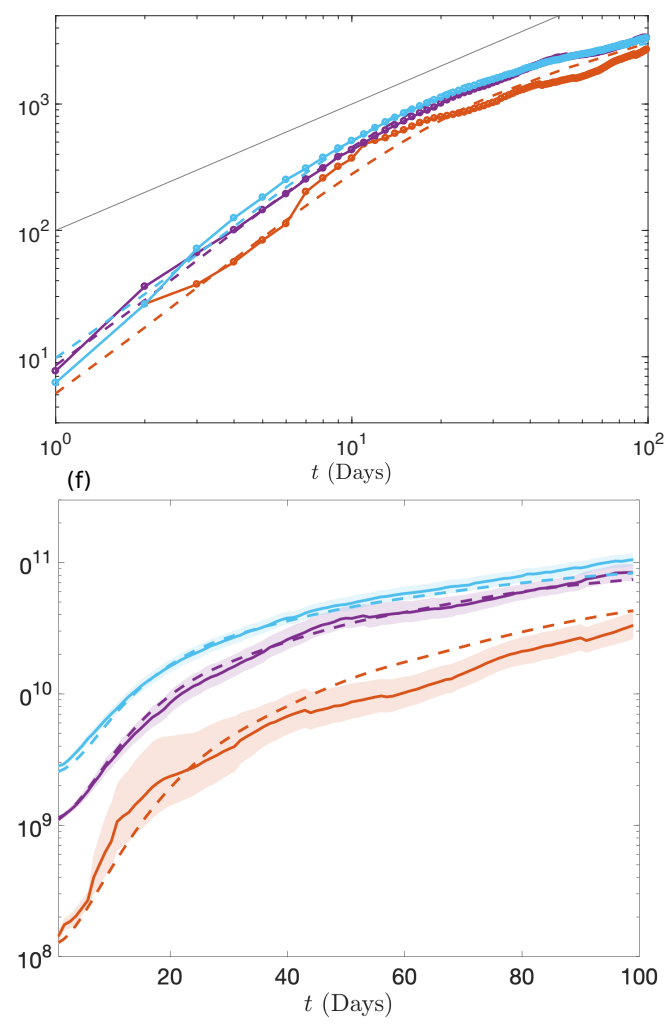

FIG. 3. Relative dispersion as a function of time for different $D_{0}$ (colors) and at different depths (a, b) for model (dashed) and RAFOS (solid) trajectories. Legend in (a) applies to figure (a) and (b). (c) and (d) show the same curves as (a) and (b) in compensated form, which would show a linear power law if the dispersion is Richardson-like. (e) and (f) show same curves as (a) and (b), but on a semi-log axis, where a straight line indicates exponential growth. 

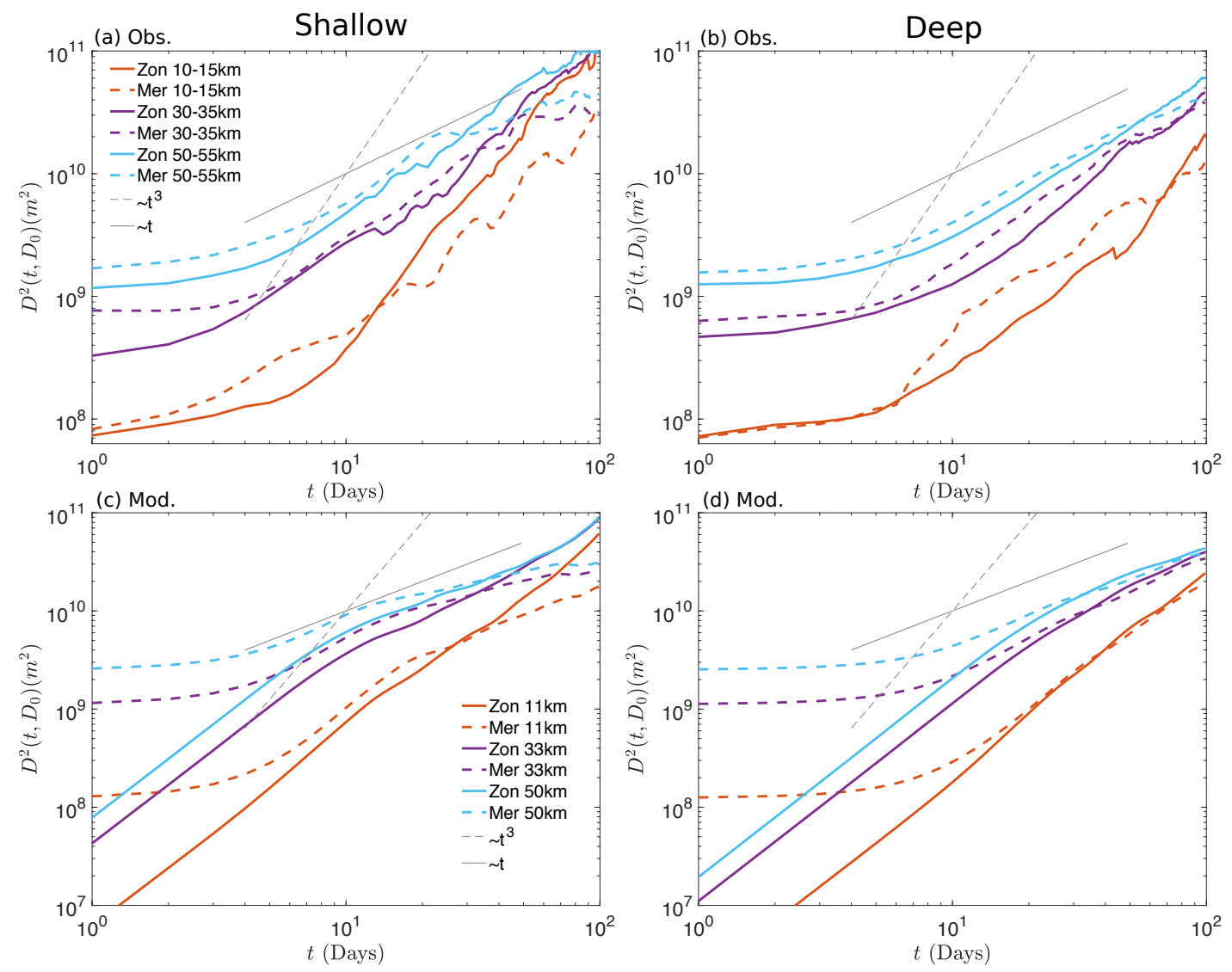

FIG. 4. Zonal (solid) and meridional (dashed) relative dispersion are shown in for RAFOS floats (a, b) and model particles (c, d). Legend in (a) applies to (a) and (b). Legend in (c) applies to (c) and (d). Thin gray lines correspond to different theoretical relations. 

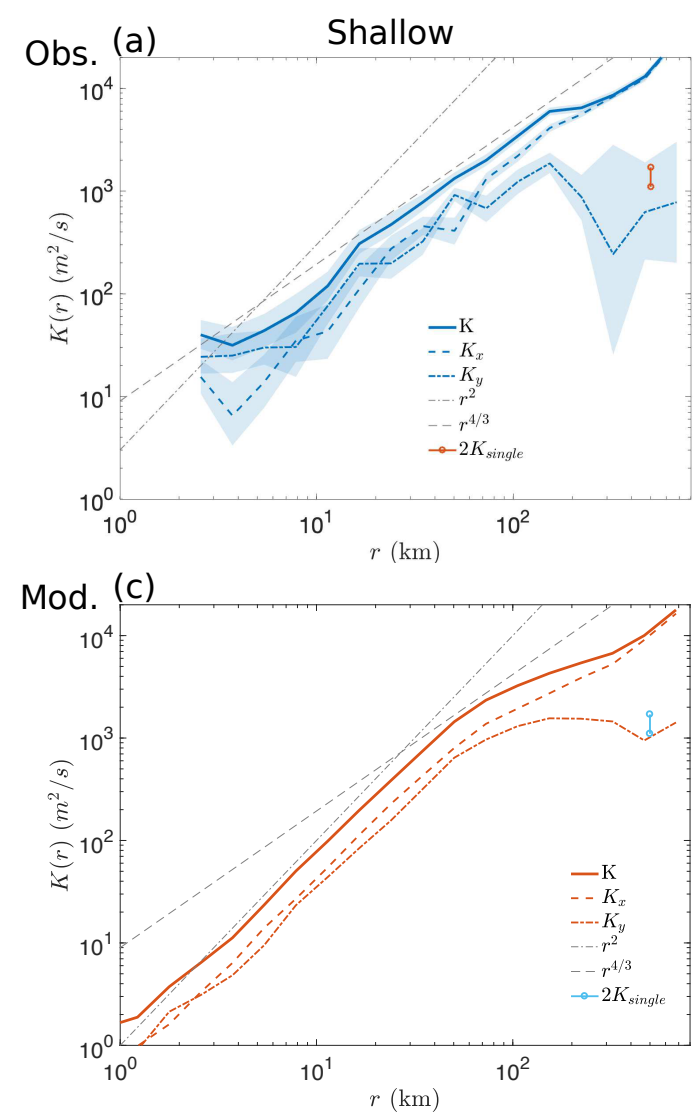

(b) Deep

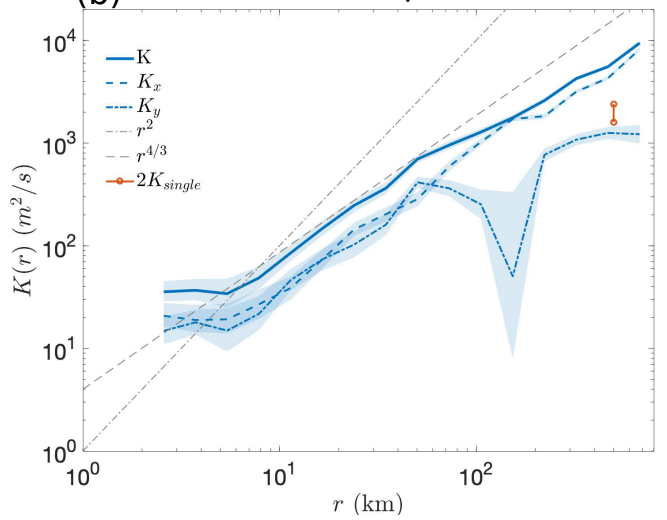

(d)

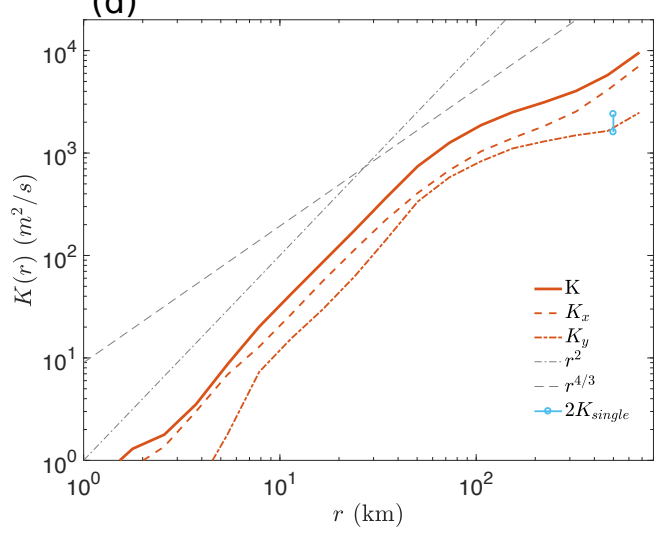

FIG. 5. Relative diffusivity as a function of separation scale for the shallow (a) and deep (b) observations, and shallow (c) and deep (d) model particles. Different colored lines correspond to the total, zonal and meridional relative dispersion, as indicated in the legend. The range of single particle diffusivities based on Balwada et al. (2016) are shown for reference.

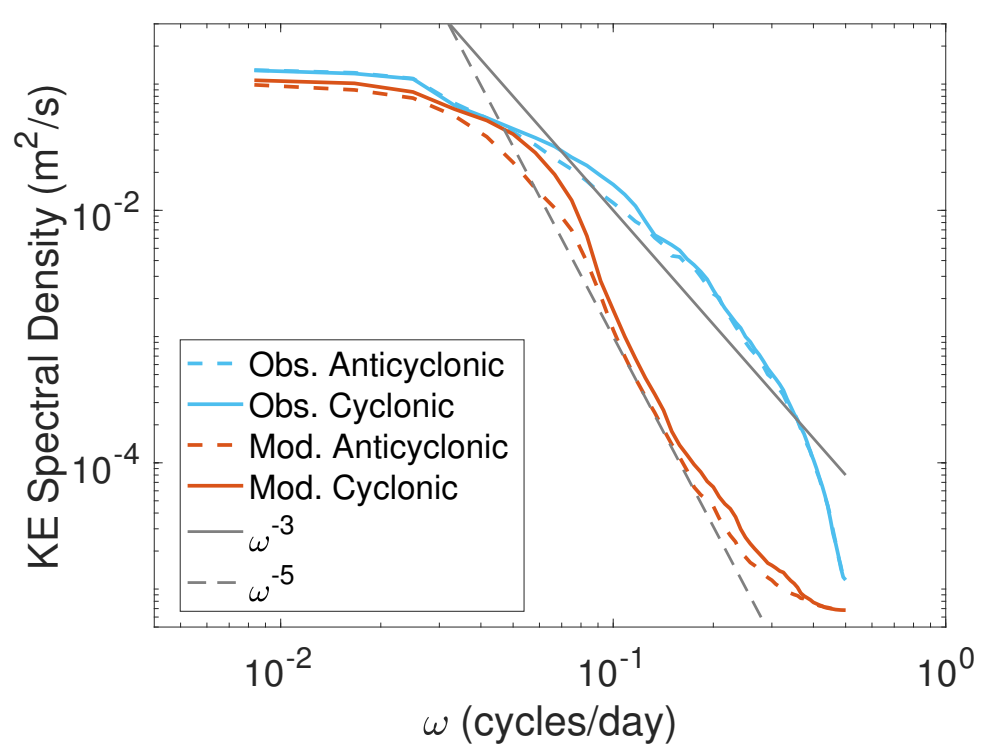

FIG. 6. Lagrangian rotary spectra of RAFOS float (red) and model particle (blue) velocities, along with indication of some generic power laws (gray lines). 

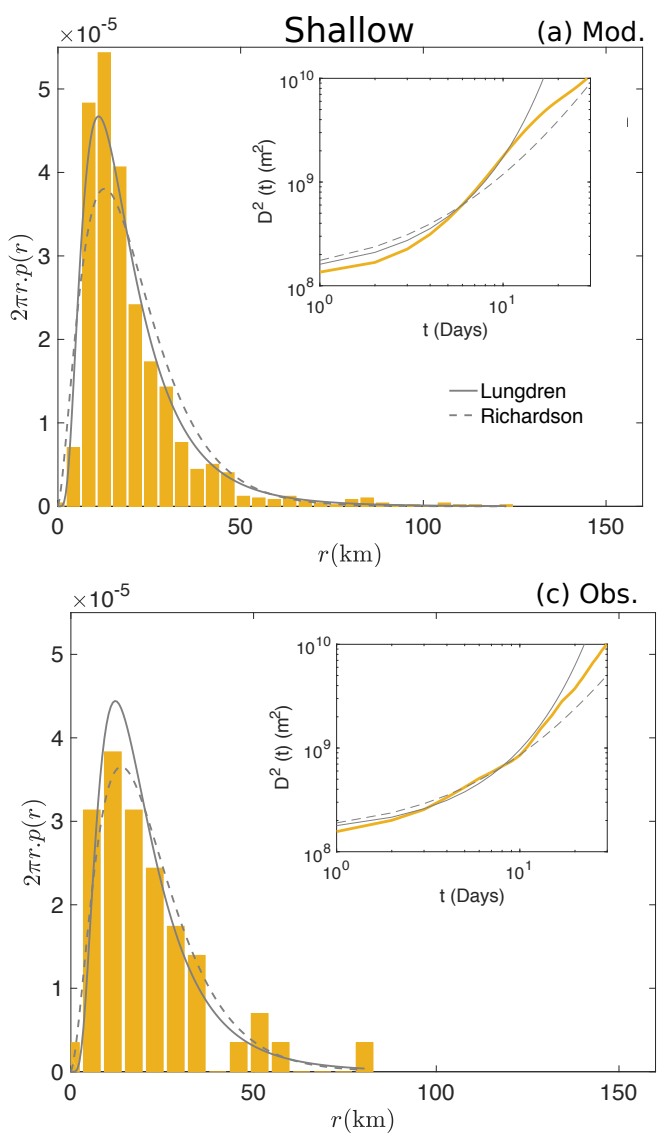
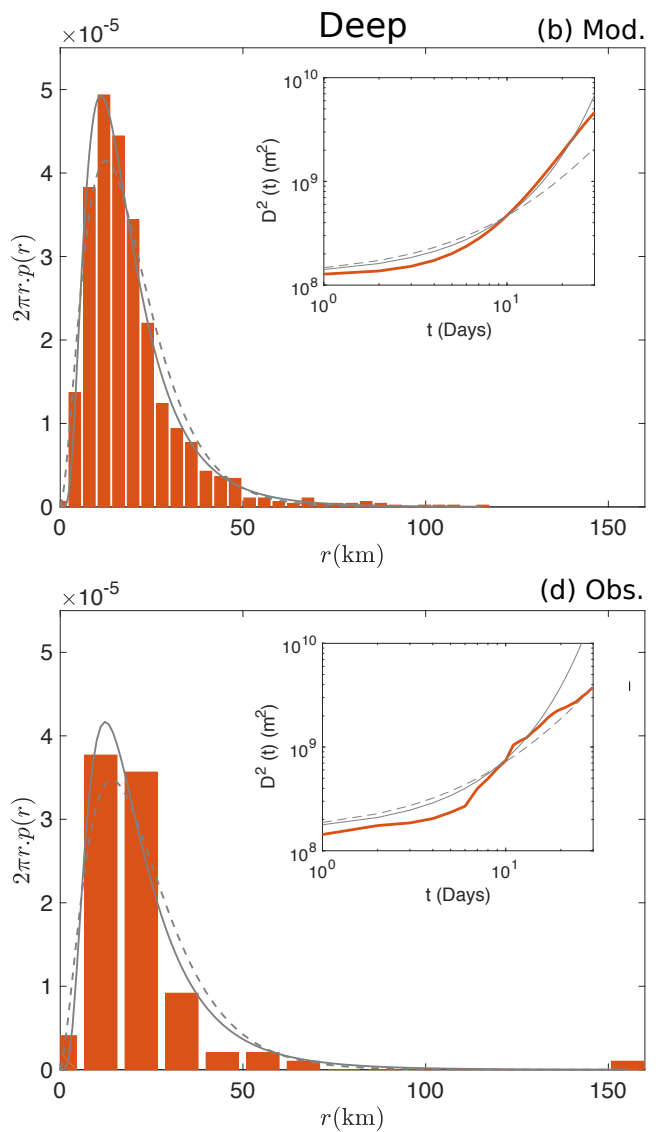

FIG. 7. Pair separation PDFs (colored bars) for the model (a,b) and DIMES observations (c,d). The left panel is for shallow depths, and the right for deep depths. The gray lines correspond to the the Lungdren and Richardson theoretical PDFs, as indicated in the legend of (a). The inset shows the corresponding relative dispersion curve, and the gray lines in the inset are the dispersion curves corresponding to the fitting parameters.
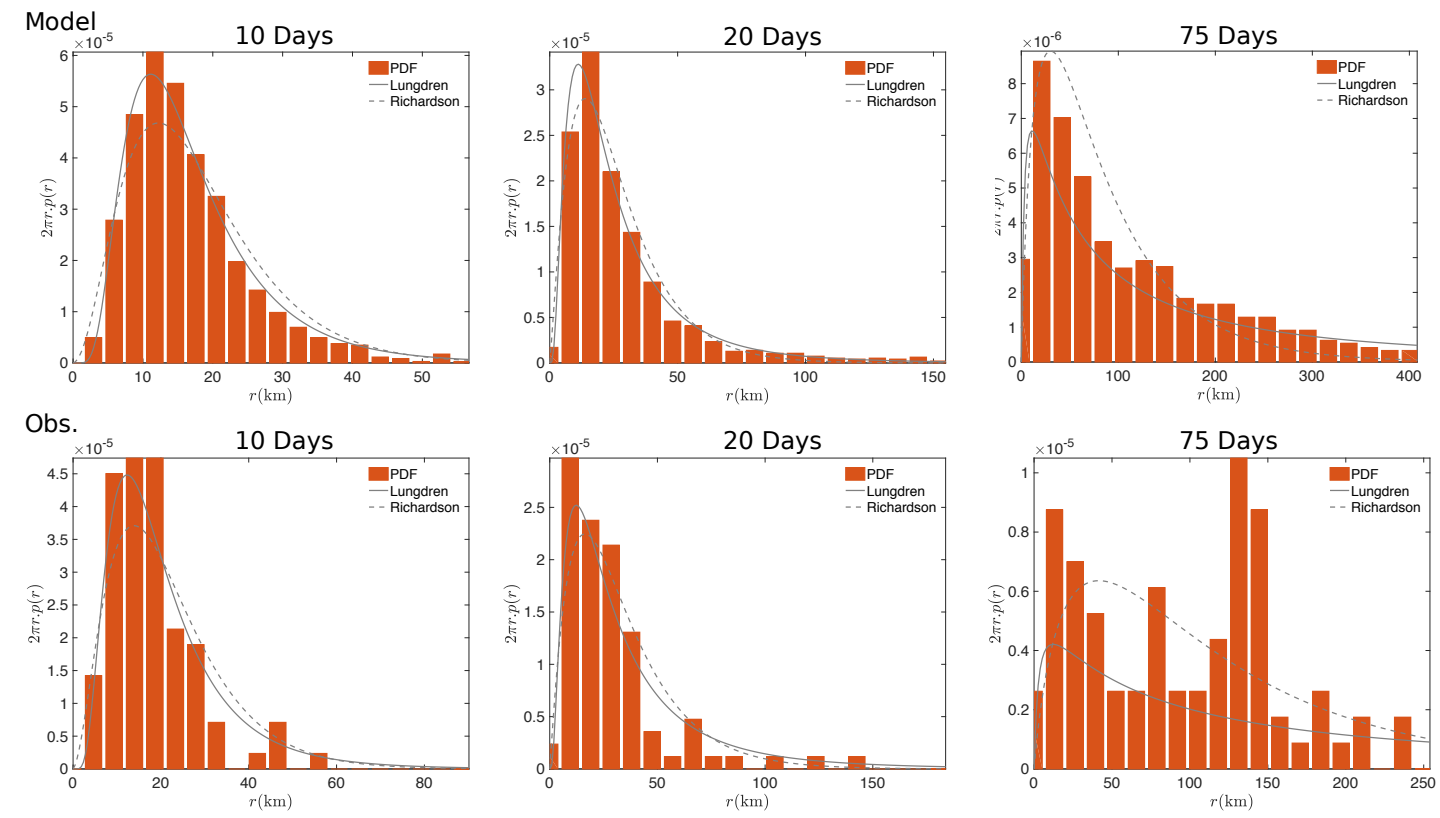

FIG. 8. Pair separation PDFs (colored lines) for the model (top row) and DIMES observations (bottom row) for the deeper data with the smallest initial pair separation at different times. The gray lines are the theoretical curves. 

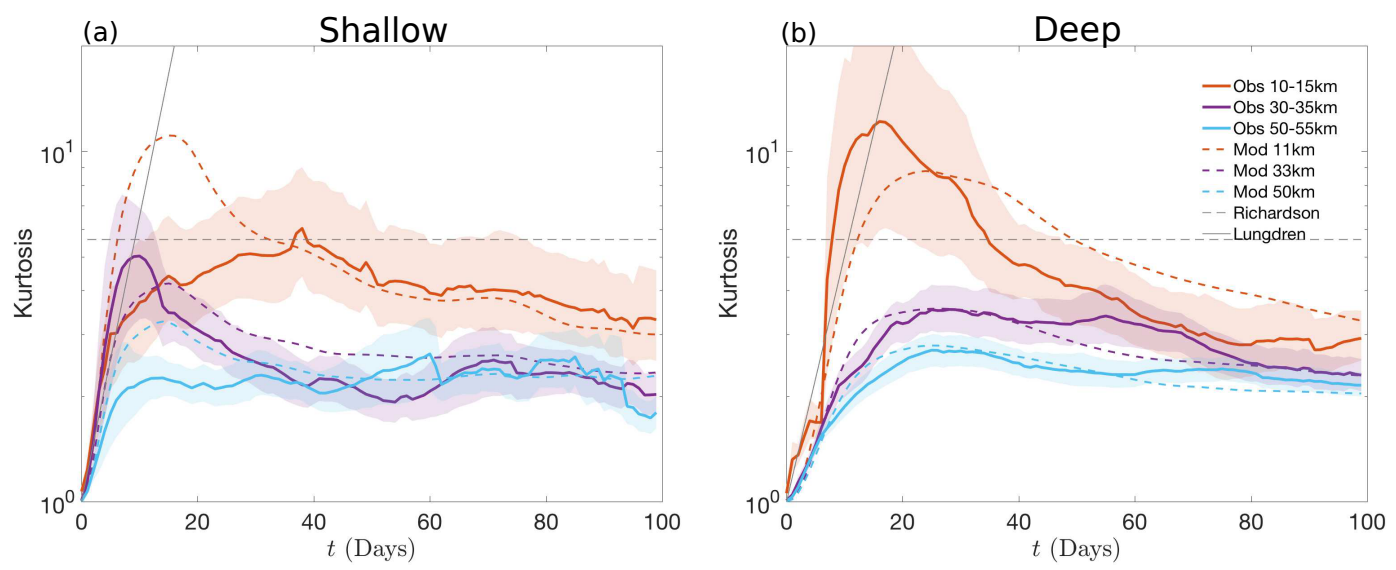

FIG. 9. Time evolution of kurtosis for model particles (dashed lines) and DIMES RAFOS floats (solid lined with colored errorbars), for different initial pair separations (colors) and at shallow (left) and deep (right) depths. The solid gray line is the theoretical kurtosis for the Lungdren/ nonlocal dispersion with the fitting parameter corresponding to the $10-15 \mathrm{~km}$ initial pair separation (Table 2). The dashed gray line indicates the value of 5.6, which is the asymptotic limit for the Richardson dispersion.
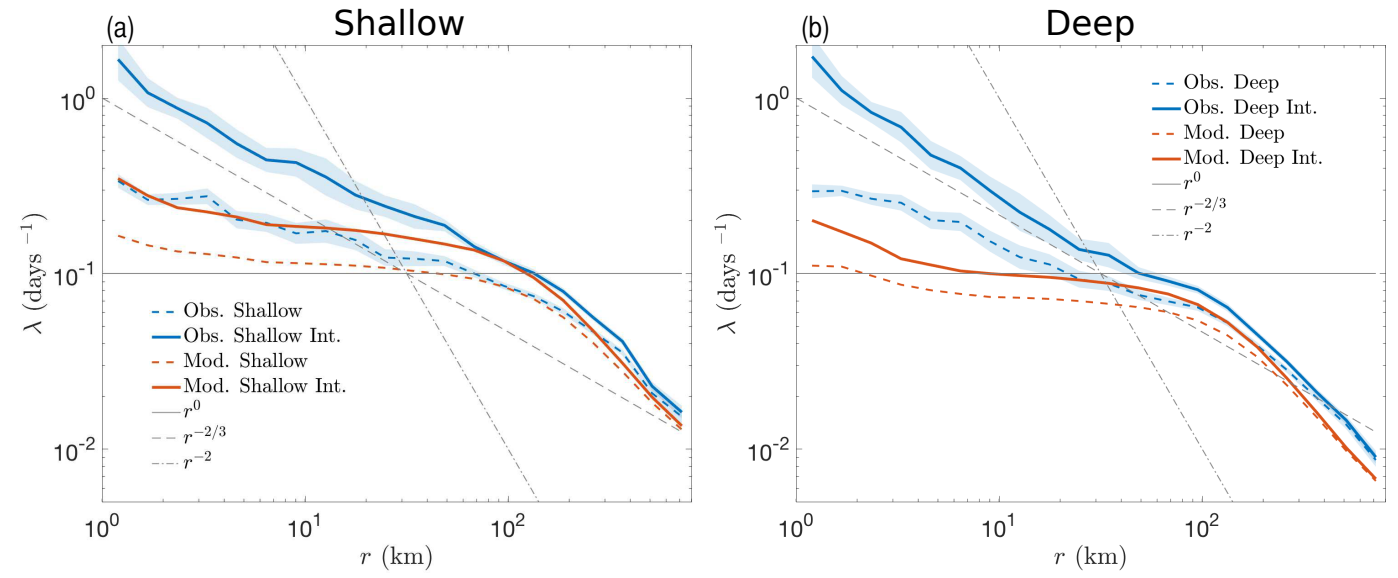

FIG. 10. Finite scale Lyapunov Exponents (FSLE) as a function of scale for the shallow (a) and deep (b) sets of trajectories from the RAFOS floats (blue) and numerical particles (red). Solid lines are the FSLEs calculated using the linearly interpolated separation time series, while the dashed lines are the FSLE without any interpolation. The dashed lines correspond to different theoretical expectations. 


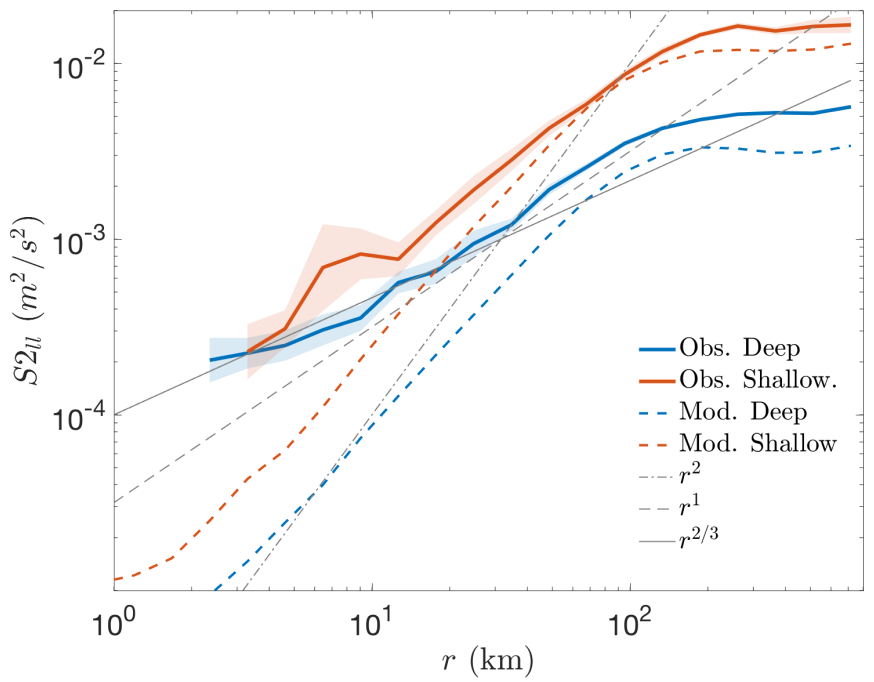

FIG. 11. Second order velocity structure functions $\left(S 2_{l l}\right)$ as a function of separation scale $(r)$ for shallow (red) and deep (blue) RAFOS floats (solid) and numerical particles (dashed). Theoretical forms are plotted as thin gray lines. 